\title{
Chiral Perturbation Theory: brief introduction *)
}

\author{
JOSÉ L. GOITY \\ Department of Phrsics, Hampton University, Hampton, VA 23668, USA, \\ and \\ Thomas Jefferson National Accelerator Facility, Newport News, VA 23606, USA.
}

In four lectures I presented a brief overview of Chiral Perturbation Theory, from its basics and some key applications in the Goldstone boson sector to its extension to the baryon sector.

\section{Introduction}

More than twenty five years ago when QCD was proposed as the fundamental theory of the strong interactions, the possibility emerged of providing a quantitative description of all strong interaction phenomena in terms of the few parameters of QCD, namely its characteristic energy scale (for which one can take a typical hadronic mass such as the mass of the $\rho$ meson), and the quark masses. While this possibility has been realized in the high energy domain where asymptotic freedom reigns and perturbative QCD can be applied, at low energies QCD is nonperturbative and confinement rules, making the connection between the physics and the QCD parameters rather remote. There are, however, aspects of QCD that transcend this non-perturbative hurdle, and these are the symmetries of QCD. In particular, one of the symmetries that results for massless quarks, chiral symmetry, seems to be approximately valid because the $u, d$, and $s$ quarks are light in comparison with the QCD characteristic scale, and even more, chiral symmetry is spontaneously broken by the QCD dynamics leading to a spectrum of light pseudoscalar mesons (pions, kaons and $\eta$ ) that are the quasi-Goldstone bosons resulting from that breaking. A situation such as this in QCD is ideal for the implementation of a low energy effective theory that in the low energy domain reproduces the physics of the full theory. Indeed, the effective theory implements the strictures of symmetries on the dynamics of the light degrees of freedom that are relerant at low energy, and this is achieved in terms of a perturbative expansion in the low energy scales (energy/momenta, and light masses) versus the characteristic scale of the full theory. The only information that the effective theory has a priori are the symmetries. The strengths of the different torms in the low energy expansion are characterized by effective parameters or low energy constants that encode the higher energy dynamics of the fundamental theory. Once these low energy constants have been determined. the effective theory is, in its range of validity, order by order in the low energy expansion equivalent to the full theory.

The effective theory of QCD is Chiral Perturbation Theory (ChPT). which was

*) Lectures given at the Xilth Indian-Summer School "Understanding the structure of hadrons". Prague (Czech Republic), August 28 - September 1, 2000. 
proposed as a full Hedged quantum field theory by Weinberg [1] and brought to its final formulation by Gasser and Leutwyler $[2,3]$, and since then it has been applied extensively to both the light pseudoscalar sector as well as the baryon sector. In both cases ChPT has brought new understanding and many predictions and has transformed the study of specific processes into precision physics. It is also interesting to mention that the connection of ChPT to QCD, that so far has happened through experimental data that allow the determination of the relewnt. low energy constants, is likely to change in the future when lattice QCD simulations with dynamical and light enough quarks become precise enough.

In this lectures I present a brief introduction to ChPT, starting with the concepts behind effective field theory, continuing with chiral symmetry in QCD, ChPT in the sector of light pseudoscalars, and finally in the sector of baryons. These lectures only cover a small fraction of the available applications of ChPT, and the interested reader can find much more in the references given in the bibliography, in particular in the more extensive lectures and reviews listed in Ref. [4].

\section{What are Effective Field Theories?}

The concept of effective field theory (EFT) sprang out from the fact that in a system or process that exhibits small ratios, a Taylor series expansion in those ratios can be implemented. One illustrative quantum mechanical example is the scattering of optical photons by neutral atoms, or Rayleigh scattering. The scale relevant to Rayleigh scattering is the typical energy splitting between the atom's excited states and its ground state (assumed Rayleigh scattering is considered on the ground state), $\Delta E \propto \alpha_{\mathrm{EM}} / a_{0}$, where $a_{0}$ is the Bohr radius. The small ratio relevant to the expansion is $\omega / \Delta E$, where $\omega$ is the photon energy. The effective theory for Rayleigh scattering can then be implemented as an expansion in this small ratio. To do this, an effective Hamiltonian $H_{\text {eff }}$ can be constructed that only contains the ground state of the atom plus the EM field. Since the atom is heavy; its recoil is negligible, and $H_{\text {eff }}$ boils down to a string of operators involving EMI fields only. Crucial in the building of $H_{\text {eff }}$ are the symmetries of the nnderlying theory: the same symmetries that are respected by the underlying theory must also be respected by the effective theory. The symmetries of electrodynamics must be, therefore, also symmetries of $H_{\mathrm{afl}}$. In particular, gauge invariance requires that the effective Hamiltonian be expressed in terms of the electric and magnetic ficlds. This together with the condition that the effective Hamiltonian has to respect parity, implies that the lowest order term has to be of the form: $\lambda_{\mathrm{E}} E^{2}+\lambda_{\mathrm{M}} B^{2}$. The EMI polarizabilities $\lambda_{E . M}$ are of order $1 / \Delta E$ and encode the higher energy physics that. is relevant to this order. while the EM fields are of $\mathcal{O}(\omega)$. Higher order terms in $H_{\text {eff }}$ are suppressed by powers of $\omega^{2} / \Delta E^{2}$. Right away one can see the power of the low energy expansion: only two parameters enter at lowest order (one of them quite small. namely $\lambda_{11}$ ), and a strict prediction about the cross section at leading order can be immediately clawn: it is proportional to $\omega^{4}$. Ref. [5] provides a mor' detailed presentation of Rayleigh scattering along the effective theory lines.

This example shows that an effective theory applies to physics processes below 

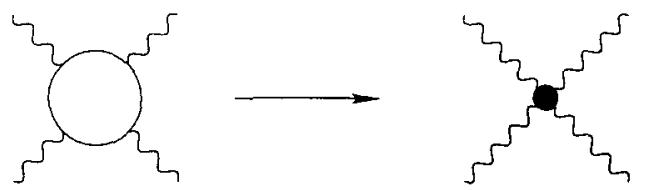

Fig. 1. From QED to the EH EFT: the diagram on the left contains an electron loop, and the one on the right represents the corresponding $\mathrm{LO}$ contact interaction in the EFT.

a characteristic scale of energy of the system. Only low energy degrees of freedom enter in the effective theory, namely those active below that characteristic scale. The degrees of freedom active above the characteristic scale do have effects at low energy, and these effects are encoded in the effective constants (e.g., the polarizabilities in Rayleigh scattering), to be called low energy constants in the following, that determine the strength of the different terms in the effective theory. And finally, the only a-priory constraints that determine the most general form of the effective theory are the symmetries of the full theory. In order to introduce EFT, let us now look at another illustrative example, namely, the Euler-Heisenberg EFT.

\subsection{Euler-Heisenberg}

The first EFT in the modern sense was formulated by Euler and Heisenberg back in 1936 [6]. This EFT describes $\gamma-\gamma$ scattering at low energy, which means below the $e^{+}-e^{-}$threshold that sets the scale for the low energy expansion. The electron field can then be integrated out leaving the photon as the only relevant degree of freedom. The interaction between photons is induced by the vacuum fluctuations of the electron field (Fig. 1), and is induced in the process of integrating the latter out. The vacuum fluctuations take place in a volume of space-time characterized by the Compton wave length $\lambda_{\mathrm{e}}$ of the electron, and such fluctuations look pointlike to photons with wave lengths much larger than $\lambda_{\mathrm{e}}$. Thus, the expansion here will be in the ratio $\lambda_{\mathbf{e}} / \lambda_{\gamma}=\lambda_{\mathrm{e} i} \dot{w}_{\gamma}$. As in the case of Rayleigh scattering, one can now use the symmetries of QED to write down the most general effective Lagrangian as an expansion in the small ratio. Gauge invariance demands that the gauge-invariant field strength tensor $F^{\prime \prime \prime}$ be used as building block. Each term in the Lagrangian will contain a low energy constant (LEC) that characterizes the strength of that term, and which in QED can be calculated perturbatively in $\alpha_{\text {EM }}$. Even in a world where the electron's charge would be large and the perturbative expansion of the LECs could not be performed, the general form of the Euler-Heisenberg Lagrangian would be the same. The EH Lagrangian is given by:

$$
\begin{aligned}
\mathcal{L}_{\mathrm{EH}}= & -\frac{1}{4} F_{m \prime} F^{\mu \prime \prime}+\frac{\alpha}{m_{\mathrm{e}}^{2}} \partial^{\rho} F_{\mu \nu} \partial_{\rho} F^{\mu \prime \prime}+\frac{\beta}{m_{\mathrm{e}}^{4}} \square F_{\mu \nu} \square F^{\prime \prime \prime} \\
& +\frac{a}{m_{\mathrm{e}}^{4}}\left(F_{\mu \nu} F^{\mu \nu}\right)^{2}+\frac{b}{m_{\mathrm{e}}^{4}}\left(\tilde{F}_{\mu \nu} F^{\mu \nu}\right)^{2} \\
& +\frac{r}{m_{\mathrm{e}}^{6}} F^{2} \square F^{2}+\cdots
\end{aligned}
$$




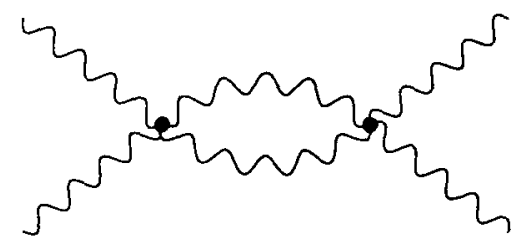

Fig. 2. Unitarity correction to $\gamma-\gamma$ scattering in the EH EFT.

Some of the terms can be disposed off by using the classical equations of motion satisfied at lowest order, namely, $\partial_{\mu} F^{\mu \nu}=0$ and $\square F^{\mu \nu}=0$. The effects of this disposal are absorbed in corrections to the remaining LEC's. If one would include the propagation of low energy electrons, such as in atoms, those terms are also important: for instance disposing of the second term introduces a contact interaction between the electron and the nucleus that is precisely the Uehling term [7].

Unitarity corrections: A full fledged QFT can now be implemented from $\mathcal{L}_{\mathrm{EH}}$. It is necessary to implement the unitarity of the S-matrix at each order in the low energy expansion. This is achieved by using ordinary Feynman rules to calculate loop corrections. The lowest order unitarity corrections stem from the diagram of Fig. 2, and the loop integrals associated with this diagram are of the form:

$$
\int \frac{\mathrm{d}^{d} k}{(2 \pi)^{d}} \frac{k_{\mu} k_{\nu}(P-k)_{\rho}(P-k)_{\sigma}}{k^{2}(P-k)^{2}} .
$$

These integrals are UV-divergent and need to be regularized. At this point it is important to introduce a regularization that preserves the symmetries of QED and does not bring in any large mass scales (which an explicit cut-off regularization would do): the natural choice satisfying these requirements is climensional regularization. The integral is of dimension four, and therefore it must be equal to a four-rank tensor containing four powers of $P$ times the integral:

$\int \frac{\mathrm{d}^{d} k}{(2 \pi)^{d}} \frac{1}{\left(k^{2}+\mathrm{i} \epsilon\right)\left((P-k)^{2}+\mathrm{i} \epsilon\right)}=\frac{1}{(4 \pi)^{2}}\left(\frac{1}{\epsilon}-\gamma_{E}+2+\log 4 \pi-\log \frac{-P^{2}}{\mu^{2}}\right)$

that displays the unitarity cut at $P^{2}=0$. Thus, unitarity introduces non-analyticities in the low energy expansion. Such non-analyticities are well dealt with by the effective theory, and reside in the loop-diagrams; the effective Lagrangian is always analytic in the low energy domain.

The vertices in Fig. 2 represent the lowest order interactions, and are proportional to $1 / m^{1}$. Therefore. the diagram is proportional to $1 / m_{\mathrm{e}}^{8}$. This readily implies that the CT infinities will be lifted by counterterms of $\mathcal{O}\left(1 / m_{\mathrm{e}}^{8}\right)$ in the effective Latgrangian. Note that in dimensional regularization the photon tadpoles vanish. Thus. the first one loop rorrections are the ones just described. The physically meaningful result for the $\gamma \gamma \rightarrow \gamma$ ? amplitude is obtained after renormalization. This is achieved 
by tuning the counterterms so that the results of the EFT match those of the full theory at the desired order.

\subsection{Low energy power counting}

The low energy expansion of the EH EFT can be ordered in powers of the small ratio $p / m_{\mathrm{e}}$ where $p$ denotes a generic small momentum. In the following a contribution of order $\left(p / m_{\mathrm{e}}\right)^{n}$ will be said to be of order $p^{n}$. The field strength tensor is counted as a quantity of $\mathcal{O}(p)$ (the EM potential $A_{\mu}$ counts here as of order $\left.\mathcal{O}\left(p^{0}\right)\right)$ and derivatives are of $\mathcal{O}(p)$. With this it is easy to order the different terms in $\mathcal{L}_{\mathrm{EH}}$ : the term $F^{2}$ is of $\mathcal{O}\left(p^{2}\right)$, the next of $\mathcal{O}\left(p^{4}\right)$, etc. On the other hand, for connected loop diagrams the low energy power counting for the integral is the naive power counting of the diagram, namely:

$$
\nu=4 L-2 I_{\gamma}+\sum_{i=1}^{V} \nu\left(v_{i}\right),
$$

where $L$ is the number of loops, $I_{\gamma}$ the number of photon propagators, $V$ is the number of vertices, and $\nu\left(v_{i}\right)$ is the order of the vertex $v_{i}$. There is a constraint given by the topological identity for a connected diagram, namely $V-I_{\gamma}+L=1$. With this it is assured that $\nu>0$ if all the vertices are of $\mathcal{O}\left(p^{2}\right)$ or higher. As illustration, the one-loop diagram just discussed has two vertices of $\mathcal{O}\left(p^{4}\right)$ and two propagators, giving $\nu=8$, as previously noticed.

\subsection{Lessons from Euler-Heisenberg}

A low energy EFT can be formulated whenever the full theory shows the following characteristics:

i) light degrees of freedom can be identified with masses much smaller than a characteristic scale $\Lambda_{\mathrm{EFT}}$, above which heavier states of the full theory become active;

ii) the small masses are natural, which means that in the context of the full theory they are protected from becoming large (such a protection can only come from (approximate) symmetries and their specific realization. For instance, in EM. gauge invariance enforces the photon to be massless ${ }^{1}$ ), and in QCD the light pions result from the spontaneously broken approximate chiral symmetry. Other symmetries that can lead to a light spectrum are (approximate) conformal symmetry and supersymmetry;

iii) the EFT is implemented by writing the most general effective Lagrangian with the light degrees of freedom and respecting the the symmetries of the full theory and by carrying out a loop expansion.

Next we put this to work for the topic of these lectures: ChPT.

1) When the Higgs mechanism occurs, although gauge invariance is a symmetry of the theory. the gauge bosons are massive, and a low energy expansion would be possible only it they are much lighter than the scale of the Higgs mechanism. This is the case when the gauge coupling is small. as in the Standard Model. 


\section{Chiral symmetry in QCD}

In QCD we can readily identify two kinds of quarks for which the physics is quite different. These are the light quarks $(u, d, s)$, with masses much smaller than the characteristic scale of $(Q C D$ that emerges from the breaking of scale invariance by the quantum theory and that we can identify as shown later with $\Lambda_{1}=4 \pi F_{\pi} / \sqrt{N_{r}}$, and heavy quarks with masses larger than $\Lambda_{\chi},(c, b, t)$. We will in the following only consider the light quark sector. The QCD Lagrangian in this sector is:

$$
\mathcal{L}_{\mathrm{QCD}}=-\frac{1}{4} G_{\mu \nu}^{a} G_{a}^{\mu \nu}+\bar{q}(\mathrm{i} \not D-\mathcal{M}) q+\frac{\theta}{(4 \pi)^{2}} G_{\mu \nu}^{a} \tilde{G}_{a}^{\mu \nu}
$$

where $G_{\mu \nu}^{a}$ is the gluon field strength tensor, $q$ is the color and flavor triplet of quarks, $D_{\mu}$ is the QCD covariant derivative, $\mathcal{M}$ is the light quark mass matrix, $\tilde{G}_{\mu \nu}$ is the dual of $G_{\mu \nu}$, and the last term (the " $\theta$-term") is strongly constrained by bounds to strong CP violation. As one can immediately check using the chiral projections of the quark fields,

$$
q_{\mathrm{L}}=\frac{1+\gamma_{5}}{2} q \text { and } \quad q_{\mathrm{R}}=\frac{1-\gamma_{5}}{2} q
$$

the interactions between quarks and gluons preserve chirality. Only the mass term connects the left- and right- handed chirality sector. Thus, in the limit of massless quarks (chiral limit), $\mathcal{L}_{\mathrm{Q} C \mathrm{D}}$ exhibits a flavor symmetry:

$$
\mathrm{SU}_{\mathrm{L}}(3) \times \mathrm{SU}_{\mathrm{R}}(3) \times \mathrm{U}_{\mathrm{A}}(1) \times \mathrm{U}_{\mathrm{B}}(1) .
$$

Here the $\mathrm{SU}_{\mathrm{L}(\mathrm{R})}(3)$ operates on $q_{\mathrm{L}(\mathrm{R})}, \mathrm{U}_{\mathrm{B}}(1)$ is the baryon number symmetry, and $\mathrm{U}_{\mathrm{A}}(1)$ corresponds to the rotations $q \rightarrow \exp \left(-\mathrm{i} \alpha \gamma_{5}\right) q$. The first two factors above are called the chiral symmetry group of QCD. The $\mathrm{U}_{\mathrm{A}}(1)$ symmetry, while a symmetry of the massless QCD Lagrangian, is not a symmetry of the full quantum theory. The quantum fluctuations of the quark fields in the gluon background lead to the explicit breaking of this symmetry at $\mathcal{O}(\hbar)$, a phenomenon known as the $\mathrm{U}_{\mathrm{A}}(1)$ anomaly. Since this breaking is proportional to the strong coupling constant $\alpha_{\mathrm{S}}$, it turns out that in a world with a large number $N_{\mathrm{c}}$ of colors the anomaly becomes of $\mathcal{O}\left(1 / N_{c}\right)$, and the symmetry tends to be restored. This limit is actually a very useful one to consider as a starting point for the EFT, but I will not elaborate further on it in these lectures.

\subsection{Spontaneous chiral symmetry breaking}

If chiral symmetry would be a symmetry of the QCD vacuum, i.e.. if it would be realized à la Wigner-Woyl. the following puzzle of parity doubling emerges. Consider the conserved axial charges $Q_{A}^{a}=\int \mathrm{d}^{3} x A_{0}^{a}(x)$, with $A_{\mu}^{a}=L_{\mu}^{a}-R_{\mu}^{a}$ where $L_{\mu}^{a}$ is the octet of currents associated with $\mathrm{SU}_{L}(3)$, etc. These charges are psendoscalar operators that in the chiral limit commute with the QCD Hamiltonian. and if $|E\rangle$ is an eigenstate with energy $E$, then the state $Q_{A}^{a}|E\rangle$ has the same energy. Since 
parity is a symmetry of $Q C D$, without loss of generality $|E\rangle$ can be taken to be of definite parity, in which case $Q_{\mathrm{A}}^{a}|E\rangle$ will be a parity eigenstate as well but with opposite parity. There are no traces of this parity doubling in the hadronic spectrum. What is the solution to this puzzle?. One possibility is that actually the axial charges are not conserved because the quark masses are not small, and one is far from the chiral limit. As made evident later in the lectures, this possibility is safely excluded. The other possibility is that the vacuum of QCD does not respect chiral symmetry, i.e., chiral symmetry is spontaneously broken (Nambu-Goldstone realization). Indeed, this is the realization that happens in QCD in the chiral limit. Apart of the phenomenological evidence that will be made clear later, there are rigorous theoretical arguments, such as the 'tHooft [8] flavor anomaly matching conditions that, because they cannot be fulfilled in QCD with two or more flavors, require spontaneous chiral symmetry breaking ( $\mathrm{S} \chi \mathrm{SB})$, and the behavior of the quark spectral function at zero momentum which can be shown to be nonvanishing even in the chiral limit [9] signaling $S \chi S B$ as well. Finally, one question is what is the pattern of $S_{\chi} \mathrm{SB}$; it can be shown [10] that in the limit where the $\theta$ angle vanishes, the vacuum state must be of even parity. This implies that the pattern is $\mathrm{SU}_{\mathrm{L}}(3) \times \mathrm{SU}_{\mathrm{R}}(3) \rightarrow \mathrm{SU}_{\mathrm{L}+\mathrm{R}}(3)$. The axial charges are generators of infinitesimal transformations in the coset $G / H=\mathrm{SU}_{\mathrm{L}}(3) \times \mathrm{SU}_{\mathrm{R}}(3) / \mathrm{SU}_{\mathrm{L}+\mathrm{R}}(3)$, and therefore do not leave the vacuum invariant. There is a manifold of degenerate vacua that can be put into a one to one correspondence with the elements of the coset. In the limit of infinite volume, it is not possible by any physical process to bring the system from one vacuum state to another. In this case, the system picks spontaneously one of the vacua and the whole physics occurs on the Hilbert space built above this vacuum. This in particular solves the parity doubling puzzle: states connected by an infinitesimal axial transformation live in different worlds! The fact that it would cost zero energy to go from one vacuum state to another (but infinite action!) implies that there must be massless excitations associated with $\mathrm{S} \chi \mathrm{SB}$. Indeed, Goldstone's theorem [11] asserts that whenever a continuous symmetry is spontaneously broken, the system must show no energy gap: in a Lorentz covariant, vacuum this means massless excitations. There is one such massless excitation, or Goldstone boson (GB), for each of the generators that do not leave the vacuum invariant. In our case, there is a GB for each axial charge, i.e., eight in total. The $J^{P}$ quantum numbers of the GBs must be the same as the ones of the axial charges, namely $0^{-}$, i.e., they must be pseudoscalars. The GBs can be classically viewed as massless excitations living in the coset $G / H$, and they provide a realization of the whole symmetry group $G[12]$. Indeed, as is well known, any element g of $G$ can be expressed as $g=\tilde{u} . \tilde{h}$ where $\tilde{\iota} \in G / H$ and $\tilde{h} \in H$. Conveniently writing:

$$
g=\left(\begin{array}{ll}
r & 0 \\
0 & l
\end{array}\right) . \quad \tilde{h}=\left(\begin{array}{ll}
h & 0 \\
0 & h
\end{array}\right), \quad \tilde{u}=\left(\begin{array}{cc}
u & 0 \\
0 & u^{\dagger}
\end{array}\right),
$$

where the block diagonal matrices are elements of SU(3), the transformation law of $u$ under a general chiral transformation $(L, R)$ is then easily obtained from the 
coset decomposition:

$$
(L, R): g=g^{\prime} \quad \Rightarrow \quad R u h=u^{\prime} h^{\prime} \text {, and } L u^{\dagger} h=u^{\prime \dagger} h^{\prime},
$$

leading to the following transformation law for $u$ :

$$
u^{\prime \dagger} R u=u^{\prime} L u^{\dagger} \text {. }
$$

Since $u$ can be used to identify an element of the coset, one can as well use $U=u^{2}$ to identify that element. Under the above transformation: $U \rightarrow R U L^{\dagger}$. The GB fields can then be identified as the coordinates of a parametrization of the $\mathrm{SU}(3)$ matrix $U$. A convenient parametrization is the exponential one:

$$
U(x)=\exp \left(-\mathrm{i} \frac{\pi^{a}(x) \lambda^{a}}{F_{0}}\right),
$$

where $\pi^{a}(x)$ is the octet of GBs $(a=1, \ldots, 8)$, which we can immediately identify with the pions, kaons and $\eta$, and $\lambda^{a}$ are the hermitian Gell-Mann matrices, which are here normalized to $\left\langle\lambda^{a} \lambda^{b}\right\rangle=2 \delta^{a b},(\langle A\rangle=\operatorname{Tr} A)$, and $F_{0}$ is a constant with dimensions of energy that is identified with the pion decay constant in the chiral limit. More explicitly:

$$
\Pi=\pi^{a} \lambda^{a}=\left[\begin{array}{ccc}
\pi^{0}+\eta / \sqrt{3} & \sqrt{2} \pi^{+} & \sqrt{2} \mathrm{~K}^{+} \\
\sqrt{2} \pi^{-} & -\pi^{0}+\eta / \sqrt{3} & \sqrt{2} \mathrm{~K}^{0} \\
\sqrt{2} \mathrm{~K}^{-} & \sqrt{2} \overline{\mathrm{K}}^{0} & -\frac{2}{\sqrt{3}} \eta
\end{array}\right]
$$

One can immediately see that the transformation properties under parity and charge conjugation operations of the pseudoscalar octet imply the following transformation laws for $U$ :

$$
P: U(\vec{x}, t)=U^{\dagger}(-\vec{x}, t) ; \quad \mathcal{C}: U=U^{T} .
$$

At this point one can see that QCD offers the perfect situation for an EFT as it has a set of naturally light states. Before proceeding to the EFT, let us introduce a useful tool, namely, external sources.

\subsection{External sources}

Most aspects of a QCD of interest in the EFT are best exhibited if one introduces color singlet external classical fields that couple to the degrees of freedom of the theory and that act as probes. Such sources of interest are the ones that couple to the currents assoriated with chiral transformations, as well as to scalat and pseudoscalar bilinears of the quark fields. They are so important because the relevant Ward identities involve such sources, and the very same Mind identities must be satisfied at the level of the EFT. The sources are included in tho QCD Lagrangian as follows ( $\theta$-term disregarded):

$$
\mathcal{L}_{\mathrm{Q} C \mathrm{D}}=-\frac{1}{4} G_{\mu \nu}^{a} G_{a}^{\prime \prime \prime}+\bar{q}(\mathrm{i} \not D-(s+\mathrm{i} p)) q,
$$


where $\mathcal{D}_{\mu}=D_{\mu}-\mathrm{i}(\not+i s \not)$. and

$$
v_{\mu}=\frac{1}{2} v_{\mu}^{a} \lambda^{\prime \prime}, \quad a_{\mu}=\frac{1}{2} a_{\mu}^{a} \lambda^{a}, \quad s=s_{0}+s^{a} \lambda^{a}, \quad p=p_{0}+p^{\prime \prime} \lambda^{a} .
$$

are the sources of interest. With these, the QCD Lagrangian has a local $\mathrm{SL}_{\mathrm{L}}(3) \times$ $\mathrm{SU}_{\mathrm{R}}(3)$ invariance. Note that the quark masses are included in the scalar source.

\section{Chiral Lagrangian}

With the external sources turned on, one can define chiral covariant derivatives that act on the unitary field $U(x)$ previously defined:

$$
\nabla_{\mu} U=\partial_{\mu} U-\mathrm{i}\left[v_{\mu}, U\right]+\mathrm{i}\left\{a_{\mu}, U\right\} .
$$

The scalar and pseudoscalar sources are put together in the following combination that transforms in the same way as the field $U$ :

$$
\chi=2 B_{0}(s+\mathrm{i} p)
$$

where $B_{0}$ has units of energy and as shown later, is given in terms of the $\bar{q} q$ condensate in the chiral limit. It is assumed to be of the order of $\Lambda_{\chi}$. With the building blocks, $U, \nabla_{\mu} U, \chi$, and their conjugates, we have all we need to write down the most general effective Lagrangian. Counting $\nabla_{\mu}$ as a quantity of $\mathcal{O}(p)$, and (as demanded by consistency in the mass formulas derived later) $\chi$ of $\mathcal{O}\left(p^{2}\right)$ (which means that the quark masses count as quantities of $\left.\mathcal{O}\left(p^{2}\right)\right)$, the effective Lagrangian consistent with the symmetries of QCD is a string of Lagrangians of increasing order:

$$
\mathcal{L}=\mathcal{L}^{(2)}+\mathcal{L}^{(4)}+\cdots
$$

Since $\langle U\rangle=1$ and $\left\langle U \nabla_{\mu} U^{\dagger}\right\rangle=0$, at $\mathcal{O}\left(p^{2}\right)$ only two terms can be written $\left.{ }^{2}\right)$ :

$$
\mathcal{L}^{(2)}=\frac{F_{0}^{2}}{4}\left[\left\langle\nabla_{\mu} U \nabla^{\mu} U^{\dagger}\right\rangle+\left\langle\chi^{\dagger} U+\chi U^{\dagger}\right\rangle\right]
$$

Only two LECs determine physics at leading order (LO): $F_{0}$ and $B_{0}$. Before discussing the next to LO (NLO). let us consider the LO predictions that are quite instructive.

\subsection{Mass formulas and ratios of quark masses}

Setting $v_{\mu}=a_{\mu}=p=0$ and $s=\mathcal{M}$, the ground state becomes $U=U_{0}^{T}=$ $\operatorname{diag}(1,1,1)$. The classical equations of motion immediately lead to the mass formulas:

$$
M_{\pi^{ \pm}}^{2}=2 \hat{m} B_{0} . \quad M_{\mathrm{K}^{ \pm}}^{2}=\left(m_{\mathrm{u}}+m_{\mathrm{S}}\right) B_{0}, \quad M_{\mathrm{K}^{0}}^{2}=M_{\overline{\mathrm{K}}^{0}}^{2}=\left(m_{\mathrm{d}}+m_{\mathrm{S}}\right) B_{0} .
$$

\footnotetext{
2) $\mathcal{L}^{(2)}$ has an extra symmetry that is absent in QCD; this is the intrinsic parity symmetry $U(\vec{x}, t) \rightarrow U(-\vec{x}, t)$. Terms that have this symmetry are called of even intrinsic parity. This symmetry can be broken at $\mathcal{O}\left(p^{4}\right)$ by the IVess-Zumino term as we show later
} 


$$
\begin{aligned}
& M_{\pi^{0}}^{2}=\left(2 m-\frac{4}{3}\left(m_{\mathrm{s}}-\dot{m}\right) \frac{\sin ^{2} \epsilon}{\cos ^{2} \epsilon}\right) B_{0}, \\
& M_{\eta}^{2}=\left(\frac{2}{3}\left(2 m_{\mathrm{s}}+\dot{m}\right)+\frac{4}{3}\left(m_{\mathrm{s}}-\hat{m}\right) \frac{\sin ^{2} \epsilon}{\cos ^{2} \epsilon}\right) B_{0},
\end{aligned}
$$

where $\hat{m}=\left(m_{\mathrm{u}}+m_{\mathrm{d}}\right) / 2$, and the meson masses have the EM contributions removed. These mass formulas lead to the Gell-Mann-Okubo relation that holds exactly when symmetry breaking is produced by on octet of $\mathrm{SU}(3)$ :

$$
2\left(M_{\mathrm{K}^{+}}^{2}+M_{\mathrm{K}^{0}}^{2}\right)+2 M_{\pi^{+}}^{2}-3\left(M_{\pi^{0}}^{2}+M_{\eta}^{2}\right)=0, \quad\left(\operatorname{Exp}:\left(230 \mathrm{MeV}^{-2}\right) .\right.
$$

The GM-O relation does not hold at NLO due to contributions to the meson masses that are quadratic in the quark masses and belong to the 27 of SU(3). The mixing angle between the $\pi^{0}$ and $\eta$ states is determined by $\mathrm{SU}(3)$ and isospin breaking and is small:

$$
\epsilon \simeq \frac{\sqrt{3}}{4} \frac{m_{\mathrm{d}}-m_{\mathrm{u}}}{m_{\mathrm{s}}-\hat{m}} .
$$

Clearly the mass formulas only allow us to determine ratios of quark masses, as the LEC $B_{0}$ is not a priory known. To extract the ratios one needs to use the physical meson masses with the EM contributions subtracted. At LO in the low energy expansion a theorem due to Dashen asserts that the EM contributions to the neutral meson masses ranish, and the ones for the charged ones satisfy: $\delta_{\gamma} M_{\mathrm{K}^{+}}^{2}=\delta_{\gamma} M_{\pi^{+}}^{2}$, which in the isospin limit is equal to $M_{\pi^{+}}^{2}-M_{\pi^{0}}^{2}$. With this, the $\mathrm{LO}$ mass ratios can be determined in a few different ways:

$$
\begin{aligned}
& \frac{\hat{m}}{m_{\mathrm{s}}+\hat{m}}=\frac{M_{\pi^{+}}^{2}}{M_{\mathrm{K}^{+}}^{2}+M_{\mathrm{K}^{0}}^{2}} \simeq \frac{1}{27}, \quad \frac{m_{\mathrm{d}}-m_{\mathrm{u}}}{2 \hat{m}}=\frac{M_{\mathrm{K}^{0}}^{2}-M_{\mathrm{K}^{+}}^{2}}{M_{\pi}^{2}} \simeq 0.29, \\
& \frac{2 m_{\mathrm{s}}+\frac{5}{2} \hat{m}}{3 \hat{m}}=\frac{M_{\eta}^{2}+M_{\pi^{0}}^{2}}{M_{\pi^{+}}^{2}} \simeq 17.5 .
\end{aligned}
$$

The first two ratios imply a value of 18.2 for the latter one. Thus, a consistent picture at LO is evident. With this the mixing angle becomes $\epsilon \simeq 0.56^{\circ}$, which is as expected quite small. As mentioned later on, despite being small, this angle plays a role in determining the rate of the $\pi^{0} \rightarrow \gamma \gamma$ decay when considered at the few percent level.

\subsection{The condensate}

The quark-antiquark condensate is obtained from the vacuum matrix element of the variation of the QCD Lagrangian with respect to source $s_{0}$. Since at LO in the low energy expansion this ought to coincide with that of $\mathcal{L}^{(2)}$, we have:

$$
\begin{aligned}
\langle\bar{q} q\rangle & =-\left\langle\frac{\delta}{\delta s_{0}} \mathcal{L}^{(2)}\right\rangle \\
& =-\left.\frac{F_{0}^{2}}{2} B_{0}\left\langle U+U^{\dagger}\right\rangle\right|_{U=U_{0}}=-3 F_{0}^{2} B_{0} .
\end{aligned}
$$


This determines $B_{0}$ in terms of the condensate and leads to the famous Gell-MamnOakes-Renner relation.

\subsection{Decay constant}

The pion decay constant $F_{0}$ can be obtained from the leptonic weak decar of the charged pion that involves the matrix element $\left\langle 0\left|A_{\mu}^{-}(x)\right| \pi^{+}, p\right\rangle$ of the axial current. Since at $\mathrm{LO} \mathcal{A}_{\mu}^{a}=-\frac{1}{2} F_{0} \partial_{\mu} \pi^{a}+\cdots$, that matrix element is equal to $\mathrm{i} \mu_{\mu} F_{0} \mathrm{e}^{-\mathrm{i} p \cdot r}$. The rate of $\pi^{+} \rightarrow \mu^{+} \nu_{\mu}$ is determined in terms of this matrix element, and after taking into account the Cabibbo mixing it leads to $F_{0} \simeq 93 \mathrm{MeV}$. A similar analysis for the corresponding decay of the $\mathrm{K}^{+}$gives $F_{0} \simeq 114 \mathrm{MeV}$. The breaking of SU(3) is thus quite important in the decay constants. This can only be accommodated by including the NLO, as shown later.

\section{$4.4 \pi-\pi$ scattering}

Among the key predictions of chiral symmetry are those for the scattering of GBs. In the chiral limit the interactions between GBs must vanish at zero momentum. This is a general consequence of SSB. The breaking of chiral symmetry by the quark masses introduce at LO modifications to the chiral limit predictions. Expressing the partial wave amplitudes in a threshold expansion: $t_{\ell}^{I}=q^{2 \ell}\left(a_{\ell}^{I}+q^{2} b_{\ell}^{I}+\cdots\right)$, where $I$ is the isospin and $\ell$ the angular momentum in the $s$-channel, and $q$ the magnitude of the relative three momentum, a low energy expansion of the threshold parameters $a_{\ell}^{I}$, etc., can be performed. At LO only S- and P-wave scattering occurs, and the so called scattering lengths are determined in terms of the pion mass and the decay constant as Weinberg showed long ago [13]:

$$
a_{0}^{0}=\frac{7}{32 \pi} \frac{M_{\pi}^{2}}{F_{0}^{2}}=0.16, \quad a_{0}^{2}=-\frac{1}{16 \pi} \frac{M_{\pi}^{2}}{F_{0}^{2}}=-0.045, \quad a_{1}^{1}=\frac{1}{24 \pi} \frac{M_{\pi}^{2}}{F_{0}^{2}}=0.030 .
$$

Comparing these results with the best current determinations from data [14]: $\left.a_{0}^{0}\right|_{\operatorname{Exp}}=0.22 \pm 0.005,\left.a_{0}^{2}\right|_{\operatorname{Exp}}=-0.0444 \pm 0.001,\left.a_{1}^{1}\right|_{\operatorname{Exp}}=0.038 \pm 0.05$, there is clear evidence for the need to go to NLO to describe the isosinglet channel.

\section{ChPT at Order $p^{4}$}

As already mentioned. extending the analysis to NLO is essential if one wants to obtain a reasonably precise picture of low energy QCD. Some observables are strictly of higher order. such as the different form factors, EM polarizabilities. D and higher partial wares in $\pi-\pi$ scattering, the $\pi^{0} \rightarrow \gamma \gamma$ decay, deviation from the GM-O relation, etc. On the other hand corrections to LO results are very important for many observables, in particular in those involving $K$ mesons as the ratio $F_{K} / F_{\pi} \simeq 1.21$, which at $L()$ must be equal to 1 , and even in the pion sector corrections to the S-rware scattering lengths are quite significant.

To introduce the XLO, it is first convenient to write down the most general $\mathcal{L}^{(1)}$ Lagrangian. This Lagrangian consists of two distinct pieces: one of even intrinsic: 
parity (EIP) and one of odd intrinsic parity (OIP) which we denote by $\mathcal{L}_{w z}$ and is known as the Wess-Zumino term. The most general Lagrangian with no redundant terms is obtained by imposing the LO equations of motion, and by using sereral SU(3) trace relations. In this way; the EIP Lagrangian has ten terms involving GBs. plus two terms with no GBs [3].

$$
\begin{aligned}
\mathcal{L}^{(4)}= & L_{1}\left\langle D_{\mu} U D^{\mu} U^{\dagger}\right\rangle+L_{2}\left\langle D_{\mu} U D_{\nu} U^{\dagger}\right\rangle\left\langle D^{\mu} U D^{\nu} U^{\dagger}\right\rangle \\
& +L_{3}\left\langle D_{\mu} U D^{\mu} U^{\dagger} D_{\nu} U D^{\nu} U^{\dagger}\right\rangle+L_{4}\left\langle D_{\mu} U D^{\mu} U^{\dagger}\right\rangle\left\langle\chi U^{\dagger}+U \chi^{\dagger}\right\rangle \\
& +L_{5}\left\langle D_{\mu} L^{\prime \prime} U^{\dagger}\left(\chi U^{\dagger}+U \chi^{\dagger}\right)\right\rangle+L_{6}\left\langle\chi U^{\dagger}+U \chi^{\dagger}\right\rangle^{2} \\
& +L_{7}\left\langle\chi^{\dagger} U-U \chi^{\dagger}\right\rangle^{2}+L_{8}\left\langle\chi U^{\dagger} \chi U^{\dagger}+U \chi^{\dagger} U \chi^{\dagger}\right\rangle \\
& +\mathrm{i} L_{9}\left\langle l_{\mu \nu} D^{\mu} U D^{\mu} U^{\dagger}+r_{\mu \nu} D^{\mu} U^{\dagger} D^{\nu} U\right\rangle+L_{10}\left\langle l_{\mu \nu} U r^{\mu \nu} U^{\dagger}\right\rangle \\
& +H_{1}\left\langle-l_{\mu \nu} l^{\mu \nu}+r_{\mu \nu} r^{\mu \nu}\right\rangle+H_{2}\left\langle\chi^{\dagger} \chi\right\rangle \\
& +\mathcal{L}_{W Z},
\end{aligned}
$$

where:

$$
\begin{aligned}
& l^{\mu \nu}=\partial^{\mu} l^{\prime \prime}-\partial^{\nu} l^{\mu}-\mathrm{i}\left[l^{\mu}, l^{\nu}\right], \quad l^{\mu}=v^{\mu}+a^{\mu} \\
& r^{\mu \nu}=\partial^{\mu} r^{\nu}-\partial^{\nu} r^{\mu}-\mathrm{i}\left[r^{\mu}, r^{\nu}\right], \quad r^{\mu}=v^{\mu}-a^{\mu} .
\end{aligned}
$$

The terms proportional to $H_{1}$ and $H_{2}$ represent contributions to vacuum polarization and to the condensate respectively, and both require renormalization. as most of the rest of the LECs. Indeed, as in Euler-Heisenberg, the unitarity and also tadpole corrections to one loop with vertices from $\mathcal{L}^{(2)}$ give rise to $\mathrm{UV}$ divergences that are precisely of $\mathcal{O}\left(p^{4}\right)$. This can be checked from (4) with vertices from $\mathcal{L}^{(2)}$ that, have $\nu\left(v_{i}\right)=2$. The terms in the EIP sector of $\mathcal{L}^{(4)}$ provide all the counterterms that are necessary to perform the renormalization. In dimensional regularization the LECs are written in terms of the renormalized ones:

$$
\begin{gathered}
L_{i}=L_{i}^{\mathrm{r}}(\mu)+\Gamma_{i} \lambda(\mu), \quad H_{i}=H_{i}^{\mathrm{r}}(\mu)+\Delta_{i} \lambda(\mu), \\
\lambda(\mu)=-\frac{1}{(4 \pi)^{2}} \frac{\mu^{-2 \epsilon}}{2}\left(\frac{1}{\epsilon}+\log 4 \pi+1-\gamma_{E}\right) .
\end{gathered}
$$

Although the coefficients $\Gamma_{i}$ and $\Delta_{i}$ can be obtained by calculating a convenient set of Green functions. Gasser and Leutwyler did this in a very elegant way using functional methods [3], obtaining:

\begin{tabular}{|c|cccccccccc|}
\hline$i$ & 1 & 2 & 3 & 4 & 5 & 6 & 7 & 8 & 9 & 10 \\
\hline$\Gamma_{i}$ & $3 / 32$ & $3 / 16$ & 0 & $1 / 8$ & $3 / 8$ & $11 / 144$ & 0 & $5 / 48$ & $1 / 4$ & $-1 / 4$ \\
\hline$\Delta_{i}$ & $-1 / 8$ & $5 / 24$ & & & & & & & & \\
\hline
\end{tabular}

The renormalized complings $L_{i}^{\mathrm{r}}(\mu)$ are here defined in the $\overline{M S}$ subtraction schene.

There are no ultrariolet divergences of OIP, as it is obvious from the fact that at LO no such terms appear. The coefficients of the Wess-Zumino term are antually 


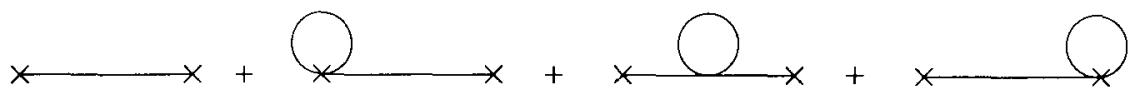

Fig. 3. The two-point function to NLO. The counter-term contributions to the currents and self energy given by the $\mathcal{L}^{(4)}$ are not explicitly shown.

fixed by the anomalies associated with the chiral transformations, as discussed in the next section.

At this point all the elements required to carry out calculations at NLO are in place. The LECs can be fixed by observables calculated to this order. As illustration of this and the improvenent brought by including NLO corrections, we discuss some of the observables already considered at LO.

\subsection{Masses and decay constants at NLO}

In order to study the masses and decay constants it is convenient to consider the two-point function of axial currents:

$$
\mathrm{II}_{\mu \nu}^{a b}(x, y)=\left\langle 0\left|T\left(A_{\mu}^{a}(x) A_{\nu}^{b}(y)\right)\right| 0\right\rangle .
$$

At NLO this two-point function is given by the diagrams in Fig. 3. At NLO we can therefore express the Fourier transform of the two-point function as follows (isospin limit considered, so that no mixing occurs):

$$
\tilde{\Pi}_{\mu \nu}^{a b}(p)=4 \mathrm{i} \delta_{a b} F_{a}^{2} \frac{1}{p^{2}-M_{a}^{2}+\mathrm{i} \epsilon} .
$$

The calculation is carried out following standard Feynman rules and dimensional regularization is used to regulate the tadpole diagrams involved. The relevant loop integral is the tadpole:

$$
\int \frac{\mathrm{d}^{d} p^{\prime}}{(2 \pi)^{2}} \frac{\mathrm{i}}{p^{\prime 2}-M_{a}^{2}+\mathrm{i} \epsilon}=\frac{1}{(4 \pi)^{2-\epsilon}} \Gamma(-1+\epsilon) M_{a}^{2(1-\epsilon)} .
$$

This gives rise to the chiral $\log$ s, i.e., terms proportional to $\log M_{a}^{2}$. With this it is straightforward to calculate the two-point function. It is easy to check that ultraviolet divergences are eliminated by the counterterms. The final results first obtained by Gasser and Leutwyler [3] are given here in the isospin limit and read:

Masses:

$$
\begin{aligned}
M_{\pi}^{2}= & 2 \hat{m} B_{0}\left(1+\mu_{\pi}-\frac{1}{3} \mu_{\eta}+2 \hat{m} K_{3}+K_{4}\right) \\
M_{\mathrm{k}}^{2}= & \left(\hat{m}+m_{\mathrm{s}}\right) B_{0}\left(1+\frac{2}{3} \mu_{\eta}+\left(\hat{m}+m_{\mathrm{s}}\right) K_{3}+K_{4}\right) \\
M_{\eta}^{2}= & \frac{2}{3}\left(\hat{m}+2 m_{\mathrm{s}}\right) B_{0}\left(1+2 \mu_{\mathrm{k}}-\frac{4}{3} \mu_{\eta}+\frac{2}{3}\left(\hat{m}+2 m_{\mathrm{s}}\right) K_{3}+K_{1}\right) \\
& +2 \hat{m}_{0}\left(-\mu_{\pi}+\frac{2}{3} \mu_{\mathrm{k}}+\frac{1}{3} \mu_{\eta}\right)+K_{5},
\end{aligned}
$$


where the chiral logs reside in

$$
\mu_{a}=\frac{1}{2 \pi^{2}} \frac{M_{a}^{2}}{\left(4 F_{\pi}\right)^{2}} \log \frac{M_{a}^{2}}{\mu^{2}},
$$

and,

Decay constants:

$$
\begin{aligned}
& F_{\pi}=F_{0}\left(1-2 \mu_{\pi}-\mu_{\mathrm{K}}+2 \hat{m} K_{6}+K_{7}\right) \\
& F_{K}=F_{0}\left(1-\frac{3}{4} \mu_{\pi}-\frac{3}{2} \mu_{\mathrm{K}}-\frac{3}{4} \mu_{\eta}+\left(\hat{m}+m_{\mathrm{s}}\right) K_{6}+K_{7}\right), \\
& F_{\eta}=F_{0}\left(1-3 \mu_{\mathrm{K}}+\frac{2}{3}\left(\hat{m}+2 m_{\mathrm{s}}\right) K_{6}+K_{7}\right) .
\end{aligned}
$$

The $K_{n}$ are given in terms of the renormalized LECs $L_{i}^{\mathrm{r}}(\mu)$ :

$$
\begin{aligned}
& K_{3}=\frac{8 B_{0}}{F_{0}^{2}}\left(2 L_{8}^{\mathrm{r}}-L_{5}^{\mathrm{r}}\right), \quad K_{4}=\left(2 \hat{m}+m_{\mathrm{s}}\right) \frac{16 B_{0}}{F_{0}^{2}}\left(2 L_{6}^{\mathrm{r}}-L_{4}^{\mathrm{r}}\right), \quad K_{6}=\frac{4 B_{0}}{F_{0}^{2}} L_{5}^{\mathrm{r}}, \\
& K_{5}=\left(m_{\mathrm{s}}-\hat{m}\right)^{2} \frac{128 B_{0}^{2}}{9 F_{0}^{2}}\left(3 L_{7}+L_{8}^{\mathrm{r}}\right), \quad K_{7}=\left(2 \hat{m}+m_{\mathrm{s}}\right) \frac{8 B_{0}}{F_{0}^{2}} L_{4}^{\mathrm{r}} .
\end{aligned}
$$

This looks complicated, but some very useful information can be readily extracted from these NLO results. The ratio $F_{\mathrm{K}} / F_{\pi}$ only involves the LEC $L_{5}^{\mathrm{r}}$, and therefore using the experimental ratio this LEC is fixed:

$$
L_{5}^{\mathrm{r}}\left(\mu=M_{\eta}\right)=2.2 \pm 0.5 \times 10^{-3}
$$

A very important result is that $F_{\eta}$ is fully determined in terms of $F_{\pi}$ and $F_{\mathrm{K}}$. Since $F_{\eta}$ cannot be directly accessed experimentally, this result can be used when studying processes where $F_{\|}$intervenes, such as the decay $\eta \rightarrow \gamma \gamma$. The GM-O relation has a deviation that can be cast in the following form:

$$
\begin{aligned}
\Delta_{\mathrm{GM}-\mathrm{O}} & =\frac{4 M_{\mathrm{K}}^{2}-M_{\pi}^{2}-3 M_{\eta}^{2}}{M I_{\eta}^{2}-M_{\pi}^{2}} \\
& =-2 \frac{4 I_{\mathrm{K}}^{2} \mu_{\mathrm{K}}-M_{\pi}^{2} \mu_{\pi}-3 M_{\eta}^{2} \mu_{\eta}}{M_{\eta}^{2}-M_{\pi}^{2}}-\frac{6}{F_{0}^{2}}\left(M_{\eta}^{2}-M_{\pi}^{2}\right)\left(12 L_{7}+6 L_{\bar{\gamma}}^{r}-L_{5}^{r}\right) .
\end{aligned}
$$

The EM corrections are small, and can be safely disregarded. Lsing the value of $L$ obtained from the decay constants one obtains the combination:

$$
2 L_{i}+L_{8}^{\mathrm{r}}\left(\mu=M_{\eta}\right)=0.3 \pm 0.4 \times 10^{-3} .
$$

Further, by considering isospin breaking it is possible to set a bound [3] on the combination

$$
\left(2 L_{\gamma}^{r}-L_{5}^{r}\right)\left(\mu=M_{\eta}\right)=0 \pm 0.5 \times 10^{-3} .
$$


At this point it is opportume to mention that some LECs are not directly ac:cessible from available experimental observables, as is the case of $L_{4}^{r}$. (On the other hand it is fortunate that some of such LECs are less relevant for a simple reason: in the large $N_{\mathrm{c}}$ limit of QCD the $L_{i}$ s with $i \neq 4,6,7$ are of $\mathcal{O}\left(N_{\mathrm{c}}\right)$, while those with $i=4,6$ are of $\mathcal{O}\left(N_{r}^{0}\right)$ (due to the OZI suppression), and $L_{7}=\mathcal{O}\left(N_{r}^{2}\right)$. Also a combination $2 L_{1}-L_{2}$ is OZI suppressed. It is thus to be expected that the OZI suppressed LECs will play a marginal role; this expectation seems to be confirmed by the phenomenology.

\subsection{Ratios of quark masses at NLO}

One interesting question is whether the LECs are determined unambiguously: from observables as done above. This translates, as shown in a moment, into whether the quark masses that appear in the effective theory must be equal to those in the QCD Lagrangian. If this would not be so, the extraction of the ratios of quark masses from the effective theory would be ambiguous. As Kaplan and Manohar noticed long ago [15], one can redefine the sources $\chi$ according to (keeping the $\theta$-term):

$$
\chi \rightarrow \chi+\lambda \exp (i \theta) \chi^{\dagger^{-1}} \operatorname{det} \chi
$$

which keeps its transformation law under chiral rotations. This transformation can be absorbed into the LECs $L_{6}, L_{7}$ and $L_{8}$. Now, the K-M transformation camnot be performed at the level of the QCD Lagrangian, so, what can prevent it from being performed in the effective theory? The answer to this question turns out. to be subtle, and involves an argument based on the large $N_{\mathrm{c}}$ behavior of the affected LECs [16]. If $\lambda$ is taken to be of order unity, then the $L_{6}$ resulting from the transformation would violate the large $N_{c}$ counting. Thus, $\lambda$ should be of $\mathcal{O}\left(N_{c}^{-1}\right)$. But this is not enough to forbid the K-M transformation altogether, however, for large $N_{\mathrm{c}}$ it can be shown that $\lambda$ must vanish to all orders in the $1 / N_{\mathrm{c}}$ expansion [16]. This should therefore settle the problem of determining the ratios of quark masses from phenomenology.

About the ratios themselves at NLO, those determined at LO are only slightly affected by the NLO corrections. On the other hand, the ratios $m_{\mathrm{u}} / m_{\mathrm{d}}$ and $m_{\mathrm{s}} / m_{\mathrm{d}}$ are related in a parameter free fashion at NLO [17]:

$$
\left(\frac{m_{\mathrm{ul}}}{m_{\mathrm{l}}}\right)^{2}+\frac{1}{Q^{2}}\left(\frac{m_{\mathrm{s}}}{m_{\mathrm{cl}}}\right)^{2}=1 .
$$

This determines an ellipse along which the ratios must lie. $Q^{2}$ is obtained from different observables. From meson masses it is given by:

$$
Q^{2}=\frac{M_{K^{2}}^{2}\left(M_{K^{2}}^{2}-M_{\pi}^{2}\right)}{M_{\pi}^{2}\left(M_{K^{0}}^{2}-M_{K^{+}}^{2}\right)} \simeq 23 .
$$

The main uncertainty is introduced by the corrections to Dashen's theorem. which until now remain controversial. The uncertainty is estimated at \pm 1.5 . Another 
determination of $Q^{2}$ rosults from the isospin breaking decay $\eta \rightarrow 3 \pi$. where the width is proportional to $Q^{-4}$, and hence a sensitive observable to determine $Q^{2}$. Here the main source of uncertainty is the experimental width. Future experiments on $\eta \rightarrow \gamma \gamma$, which is used to calibrate the partial width of interest, will most likely. allow for a more precise determination of $Q^{2}$. With the current uncertainty on $Q^{2}$ and the ratio

$$
\frac{m_{\mathrm{s}}}{m_{\mathrm{al}}}=\frac{M_{\mathrm{K}^{0}}^{2}+M_{\mathrm{K}^{+}}^{2}-M_{\pi^{+}}^{2}}{M_{\mathrm{K}^{0}}^{2}-M_{\mathrm{K}^{+}}^{2}+M_{\pi^{+}}^{2}} \simeq 20,
$$

one can give the estimate $m_{\mathrm{u}} / m_{d} \simeq 0.5$. This standard analysis shows that this ratio is larger than zero [17], excluding the possibility of a massless $u$-(quark, so much desired as a quick solution to the strong CP problem.

\subsection{Form factors}

At LO the GBs are pointlike. First at NLO their finite size is noticed through the different form factors, for instance those associated with the vector currents which we briefly present here. In order to extract the form factors one can study the three-point functions involving two axial currents and the vector current of interest. The diagrams involved are shown in Fig. 4. The EM charge radii of the $\pi^{+}$and the kaons can be expressed in terms of only one LEC, namely $L_{9}$ and
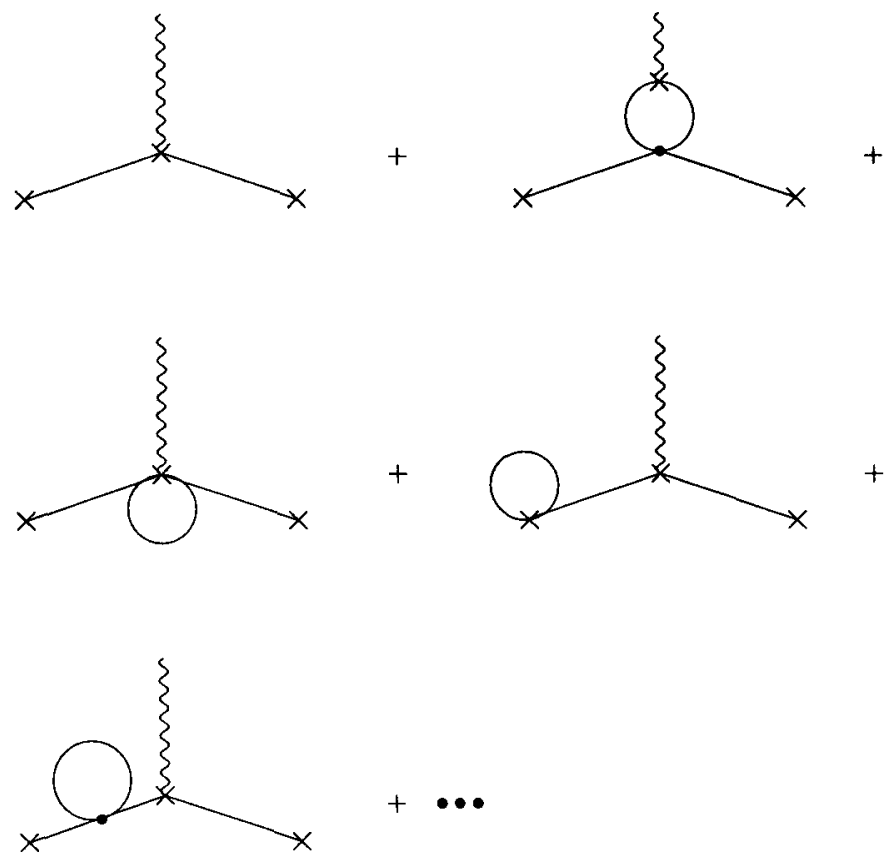

Fig. 4. The three-point function to XLO. The $\cdots$ indicate the contributions from counterterms from $\mathcal{L}^{(4)}$ 
non-analytic pieces stemming from the loop. Explicit calculation gives:

$$
\begin{aligned}
\left\langle r^{2}\right\rangle_{\gamma}^{\pi} & =\frac{12 L_{9}^{\mathrm{r}}}{F_{0}^{2}}-\frac{1}{32 \pi^{2} F_{0}^{2}}\left(2 \log \frac{M_{\pi}^{2}}{\mu^{2}}+\log \frac{M_{\mathrm{K}}^{2}}{\mu^{2}}+3\right), \\
\left\langle r^{2}\right\rangle_{\gamma}^{\mathrm{K}^{0}} & =-\frac{1}{32 \pi^{2} F_{0}^{2 \cdot}} \log \frac{M_{\mathrm{K}}^{2}}{M_{\pi}^{2}}, \\
\left\langle r^{2}\right\rangle_{\gamma}^{\mathrm{K}^{+}} & =\left\langle r^{2}\right\rangle_{\gamma}^{\pi}+\left\langle r^{2}\right\rangle_{\gamma}^{\mathrm{K}^{0}} .
\end{aligned}
$$

The mean square charge radius of the pion diverges logarithmically in the chiral limit. However, in the real world the non-analytic pieces are smaller than the term due to the counterterm and proportional to $L_{9}$. Indeed, the experimental value $\left.\left\langle r^{2}\right\rangle_{\gamma}^{\pi}\right|_{\text {Exp }}=0.44 \pm 0.03 \mathrm{fm}^{2}$ implies that $L_{9}^{\mathrm{r}}\left(\mu=M_{\eta}\right)=7.4 \pm 0.7 \times 10^{-3}$. With this the other radii are fixed, and the agreement with the experimental values is fine, although the $\mathrm{K}^{0}$ radius is rather poorly determined. One important point to be emphasized here is that the vector meson dominance model by which the form factor of the pion is clominated by the $\rho$ meson is in good agreement. This means that the value of $L_{9}$ is to a large extent determined by the existence of the $\rho$ meson. Indeed, the idea that one can identify heavier states that to a large extent determine the values of the LECs in $\mathcal{L}^{(4)}$ works remarkably well [18]. Other form factors that can be studied are the ones involved in weak semileptonic decays $\left(\mathrm{K} \rightarrow \pi \ell \bar{\nu}_{\ell}\right.$ ), for which ChPT at $\mathcal{O}\left(p^{4}\right)$ gives a good description [19].

Another form factor of great interest is the scalar form factor of the pion, which can be extracted from $\pi-\pi$ scattering. The interesting point here is that the scalar radius of the pion is substantially larger than the charge radius. For the details see Ref. [19].

\section{$5.4 \pi-\pi$ scattering at NLO}

At NLO, $\pi-\pi$ scattering involves the consideration of the four-point function of axial currents. Since one is here considering the non-strange sector, the analysis is customarily made within $S U(2)$. The LECs in $\mathrm{SU}(2)$ are related to those in SU(3) [3]. At NLO there are eight scattering parameters, namely, the two $S$-wave scattering lengths and the associated slope parameters $b_{0}^{I}$, the P-wave scattering length and the slope parameter. and two D-wave scattering lengths. There are only three NLO LECs, one of them only affecting $a_{0}^{0}$. There is therefore a great, level of predictivity that ChPT an provide.

There has been impressive recent progress in the analysis of $\pi-\pi$ scattering by using the phenomenological description of the available data by means of the Roy equations [20] (that implement the strictures of unitarity and crossing) in combination with ChPT at NLO (and even NNLO) $[21,22]$. The analysis of the arailable data in this framework leads to rather precise predictions for all the scattering parameters mentioned above. and in particular for the two S-wave scattering lengths. The NLO results are for the S-wave scattering lengths are:

$$
a_{0}^{0}=0.219 . \quad a_{0}^{2}=-0.0446, \quad 2 a_{0}^{0}-5 a_{0}^{2}=0.662 .
$$


These predictions are only slightly changed when the NNLO is implemented [22. 23]. Although most of the data available until recently was rather old. the BNL experiment on $K_{a 4}$ decay has recently given a ten-fold improvement to the data base that allows to determine the combination of phase shifts $\delta=\delta_{0}^{0}-\delta_{1}^{1}$ [24]. One. further very important experiment in the works is DIRAC [25], which will study pionium, and provide a measurement of the combination $\left(a_{0}^{0}-a_{0}^{2}\right)^{2}$. This together with the combination $\left(2 a_{0}^{0}-5\left(a_{0}^{2}\right)\right.$ that satisfies the Olsson sum rule, can load to at stringent test of ChPT through the analysis of the data without the input of ChPT. For an overview of the status of $\pi-\pi$ scattering and a list of references see Ref. [14].

\section{Anomalies and the Wess-Zumino term}

As mentioned before, in the presence of the external sources the QCD Lagrangian is invariant under local $\mathrm{SU}_{\mathrm{L}}(3) \times \mathrm{SU}_{\mathrm{R}}(3) \times \mathrm{U}_{\mathrm{A}}(1) \times \mathrm{U}_{\mathrm{B}}(1)$ (we assume here that, we add vector and pseudovector fields coupling to the two $\mathrm{U}(1)$ currents). This symmetry, however, is not the symmetry of the full quantum theory. The vacuum fluctuations of the quarks induce explicit breaking of part of the symmetry group. This phenomenon is known as anomaly [26], and plays a crucial role in determining the OIP piece of the effective Lagrangian. The anomaly emerges from the need of defining the determinant of the Dirac operator in $\mathcal{L}_{\mathrm{QCD}}$, and which results from integrating-out the quark fields. In defining the determinant of the Dirac operator an ultra-violet regularization must be introduced, and this cannot be done without spoiling some of the symmetries [27]. Of course, there is ambiguity in the way $\operatorname{det} \mathcal{D}$ is defined, and it has to do with what symmetries one chooses to preserve. This ambiguity is manifested in the possibility of adding to $\log \operatorname{det} \mathcal{D}$ local counterterms. For instance, one can judiciously add these counterterms in such a way that $\log \operatorname{det} \mathcal{D}$ is invariant under vector transformations. In this case, under an infinitesimal axial-vector transformation, $L=1+\mathrm{i} \beta$ and $R=1-\mathrm{i} \beta$, one obtains [2]:

$$
\begin{aligned}
\delta_{\beta} \log \operatorname{det} \mathcal{D}= & \frac{\mathrm{i}}{(4 \pi)^{2}} \int \mathrm{d}^{4} x\left(G \tilde{G}\langle\beta\rangle+N_{\mathrm{c}}\langle\beta \Omega\rangle\right), \\
G \tilde{G}= & \epsilon_{\mu \nu \rho \sigma} G_{a}^{\mu \nu} G_{a}^{\rho \sigma}, \\
\Omega= & \epsilon_{\mu \nu \rho \sigma}\left(v^{\mu \prime \prime} v^{\rho \sigma}+\frac{4}{3} \nabla^{\mu} a^{\nu} \nabla^{\rho} a^{\sigma}+\frac{2}{3} \mathrm{i}\left\{v^{\mu \prime \prime}, a^{\rho} a^{\sigma}\right\}\right. \\
& \left.+\frac{8}{3} \mathrm{i} a^{\prime \prime} v^{\mu \prime \prime} a^{\sigma}+\frac{4}{3} a^{\mu} a^{\nu} a^{\prime} a^{\sigma}\right), \\
\Gamma_{\mu} a_{\mu}= & \partial_{\mu} a_{\mu}-\mathrm{i}\left[v_{\mu}, a_{\nu}\right] .
\end{aligned}
$$

The coefficient in front of $\langle 3\rangle$ is the $\mathrm{L}_{A}(1)$ anomaly responsible for the explicit breaking of this symmetry. and the term $N_{\mathrm{c}} \Omega$ is the non-Abelian anomaly: The anomalies are independent of the scalar and psendoscalar sources. Since $\delta_{3}$ log det $D$ is a contribution to the rariation of the effective action with quarks integrated out. the effect of the anomalies on the Ward identities can be derived in the standard 
fashion.

The important question is now: what are the consequences of the anomalies when symmetries affected by them are spontaneously broken? This question was addressed and answered thirty years ago by Wess and Zumino [28]. They showed how to construct an effective Lagrangian that implements the anomalies in the Ward identities. Using that the variation of the effective action under an infinitesimal transformation is given by:

$$
\delta_{3} \Gamma_{\mathrm{WZ}}=\mathrm{i} \delta_{\beta} \log \operatorname{det} \mathcal{D}=-\int \mathrm{d}^{4} x\langle\beta \Omega\rangle
$$

this equation can be integrated by imposing an appropriate boundary condition. To have parity invariance, this condition must be $\Gamma_{\mathrm{WZ}}\left(v_{\mu}, a_{\mu}, U=1\right)=0$. The solution is then:

$$
\Gamma_{\mathrm{WZ}}\left(v_{\mu}, a_{\mu}, U\right)=\int \mathrm{d}^{4} x \mathcal{L}_{\mathrm{WZ}}=\sum_{n=0}^{\infty} \frac{1}{(n+1) !} \int \mathrm{d}^{4} x\left\langle\beta(x) \delta_{\beta}^{n} \Omega(x)\right\rangle,
$$

where $\beta=-\mathrm{i} \log U$, and $\delta_{\beta} \Omega$ is the linear term of the gauge transformation on $\Omega$ evaluated at $\beta$. A close form can be given for the effective action [29], which we do not display here for the sake of brevity. Clearly, $\mathcal{L}_{\mathrm{Wz}}$ breaks the intrinsic parity symmetry.

It is immediate to extract a number of processes that at LO proceed only through the WZ-term. The most well known is $\pi^{0} \rightarrow \gamma \gamma$, which is obtained by setting $a_{\mu}=0$ and $v_{\mu}=v_{\mathrm{EM} \mu}$. The amplitudes for this decay and $\eta \rightarrow \gamma \gamma$ are then given by:

$$
A_{\pi^{0}(\eta) \rightarrow \gamma \gamma}=\frac{N_{\mathrm{c}} \alpha_{\mathrm{EM}}}{(4 \pi)^{2} 2 F_{\pi_{0}(\eta)}} \frac{1}{3}\left(\frac{1}{3 \sqrt{3}}\right) F \tilde{F}
$$

leading to the widths: $\Gamma\left(\pi^{0} \rightarrow \gamma \gamma\right) \simeq 7.6 \mathrm{eV}$, and $\Gamma(\eta \rightarrow \gamma \gamma) \simeq 42 \mathrm{eV}$, to be respectively compared with the experimental ones: $7.7 \pm 0.6 \mathrm{eV}$ and $421 \mathrm{eV}$. In the latter case the large discrepancy is understood as due to the mixing between the $\eta$ and $\eta^{\prime}$. In the case of the $\pi^{0}$ the experimental situation will improve with a new measurement using the Primakoff effect [30]. A precision of slightly more than $1 \%$ in the width is expected. At this level of precision the chief correction needed to be included is due to isospin breaking [31,32] and is manifested in the mixing of the $\pi^{0}$ with the $\eta$ meson (also the $\eta^{\prime}$ if this is explicitly included in the effective theory).

One important point to be emphasized is the NLO corrections involving the WZ-term, and which correspond to going to $\mathcal{O}\left(p^{6}\right)$ in the chiral expansion. Explicit evaluation [33] shows that, neglecting mixings, the one-loop contributions to the amplitudes are absorbed by replacing the LO decay constants by the NLO ones. Indeed, this can be seen as a consequence of the non-renormalization theorem of Adler and Bardeen for anomalies. Certainly, there is plenty more interesting physics that can be further extracted from the IIZ-term $(\eta \rightarrow \pi \pi \gamma, \mathrm{K} \rightarrow \pi \pi(\bar{\nu},-\pi \rightarrow \pi \pi$. processes with virtual photons. etc). but unfortunately no space is left here to address them. 


\section{Other applications in the GB sector}

There are multiple applications of ChPT in the meson sector that we camnot cover here. The weak semileptonic decays have been exhaustively analyzed [19]. The EM interactions, in particular of the pions, have also been studied in clctail. ()f particular interest is low energy Compton scattering from where the electric and magnetic polarizabilities are extracted, and for which ChPT provides a parameter free prediction for both the charged and neutral pion [34], as well as for the kaons. The different experimental determinations of the pion polarizabilities are in disagreement, and we will have to wait until these predictions are tested. Another arena where ChPT has allowed very important progress is in weak non-leptonic: processes. This physics requires the introduction of more terms in the effective theory to describe the underlying dimension-six $|\Delta s|=1$ operators [35]. This sector has been analyzed at NLO [36], in particular the $\mathrm{K} \rightarrow 2 \pi$ and $\mathrm{K} \rightarrow 3 \pi$ decays. Also in this sector one low energy theorem was established for the decay $\mathrm{K}_{\mathrm{S}} \rightarrow \gamma \gamma$ (a process that first proceeds at the one-loop level), which states that, at the LO of this process, the amplitude is fixed in terms of the $\mathrm{K} \rightarrow 2 \pi$ amplitudes [37]. The study of rare decays has also benefitted from the use of ChPT. The current status of weak decays can be found in [38]. Finally, an interesting development is the combination of ChPT with dispersion relations [39], that with further experimental input (involving the imaginary parts of the corresponding amplitudes) and the strictures imposed by ChPT extends in a rigorous way the range of applicability of the effective theory. This has been a very active direction of work recently.

\section{Baryons}

ChPT in baryons is perhaps the most important work in progress in low energy QCD. This encompasses the study of processes involving one baryon as well as few baryons, the latter being a rather recent and exciting development.

In the lectures I addressed only the sector with one stable baryon. In this sector the implementation of the EFT can be carried out in a couple of alternative ways. One is the so called heary baryon ChPT (HBChPT) [40], and the other is the covariant baryon ChPT (CBChPT) [41, 42]. Also, there are alternative formulations in which the spin $3 / 2$ baryons are either integrated out or are kept as explicit rlegrees of freedom of the EFT. In general the situation is far less straightforward than in the GB sector. One issue that represents a potential problem is that of convergence of the low energy expansion. especially when the strange sector is considered.

In the following I will describe the basics of the mentioned approaches and will discuss a few illustrative examples.

\subsection{Chiral symmetry and baryons}

In order to formulate the EFT for baryons it is required first to have transformation laws for the baryon ficlds under chiral transformations. The spin $1 / 2$ barvon fields must be in an irrelurible representation of the group $H=\mathrm{SC}(3)$. and this rep- 
resentation depends on $\lambda_{c}$; for $N_{c}=3$ the spin $1 / 2$ baryons are in an octet, while the spin $3 / 2$ baryons are in a decouplet. In the following only the spin $1 / 2$ sector is considered. Under a chiral transformation $(L, R)$ the baryon octet transforms as follows [12]:

$$
B \rightarrow h^{\dagger}(u, L, R) B h(u, L, R), \quad B=\frac{1}{\sqrt{2}} B^{a} \lambda^{a},
$$

where the $B^{a}$ are Dirac spinors, $u=\sqrt{U}$ is given in terms of the GB fields, and $h(u, L, R)$ is as given in (8). This is a non-linear realization of the chiral group on the baryons. A covariant derivative that keeps that transformation law can be immediately defined:

$$
\begin{aligned}
\nabla_{\mu} B & =\partial_{\mu} B-\mathrm{i}\left[\Gamma_{\mu}, B\right] \\
\Gamma_{\mu} & =\frac{1}{2}\left(u^{\dagger}\left(\partial_{\mu}-\mathrm{i} r_{\mu}\right) u+u\left(\partial_{\mu}-\mathrm{i} l_{\mu}\right) u^{\dagger}\right),
\end{aligned}
$$

where the sources are $r_{\mu}=\left(v_{\mu}+a_{\mu}\right) / 2$, etc. Other building blocks with the same chiral transformation properties are the pseudoscalar

$$
\omega_{\mu}=\frac{1}{2} \mathrm{i}\left(u^{\dagger}\left(\partial_{\mu}-\mathrm{i} r_{\mu}\right) u-u\left(\partial_{\mu}-\mathrm{i} l_{\mu}\right) u^{\dagger}\right)
$$

and

$$
\chi_{ \pm}=u^{\dagger} \chi u^{\dagger} \pm u \chi^{\dagger} u
$$

It is now straightforward to to write down the effective Lagrangian for the case where a single baryon propagates. One issue is the ordering of terms according to the low energy power counting. This is because by Lorentz invariance, each term in the Lagrangian has a leading order plus terms of subleading order. Thus, when writing down manifestly Lorentz invariant Lagrangian, one may assign to a term its leading order counting. In the $\mathrm{HBChPT}$ we will see that each term has a definite counting. As usual, one can simplify the Lagrangian by use of the LO equations of motion, and several SU(3) and Clifford algebra identities [43]. The lowest order lagrangian is of $\mathcal{O}(p)$ and reads:

$$
\begin{aligned}
\mathcal{L}_{B}^{(1)}= & \langle\bar{B}(\mathrm{i} \not \bar{X}-\stackrel{o}{m}) B\rangle \\
& +D\left\langle\bar{B} \gamma^{\mu} \gamma_{5}\left\{\omega_{\mu}, B\right\}\right\rangle+F\left\langle\bar{B} \gamma^{\mu} \gamma_{5}\left[\omega_{\mu}, B\right]\right\rangle
\end{aligned}
$$

Here $\stackrel{o}{m}$ represents the common baryon masses in the chiral limit. Note that the mentioned counting holds for the Dirac operator i $\not \nabla-\stackrel{o}{m}$, although the mass term and the kinetic term are both of $\mathcal{O}\left(p^{0}\right)$. There is no chiral symmetry breaking by the quark masses at LO. They enter at in the $\mathcal{O}\left(p^{2}\right)$ Lagrangian:

$$
\mathcal{L}^{(2)}=b_{\mathrm{D}}\left\langle\vec{B}\left\{\chi_{+} \cdot B\right\}\right\rangle+b_{\mathrm{F}}\left\langle\bar{B}\left[\chi_{+}, B\right]\right\rangle+b_{0}\left\langle\chi_{+}\right\rangle\langle\bar{B} B\rangle+\cdots .
$$

where the $\cdots$ contain other terms involving $\omega$, etc. For the complete expressions in the case of two flavors see Ref. [44], and for three flavors see Ref. [45]. In a similar 
fashion one can proceed to the construction of the higher order Lagrangians. As it is already apparent, the chief distinguishing feature of the low energy expansions between the GB and baryon sectors is that in the latter the expansion proceeds in steps of one unit in the power comnting instead of two. This makes the baryon secton much more difficult to study, especially concerning the question of convergence of the expansion. Currently people have ventured as far as the $\mathcal{O}\left(p^{4}\right)$ (in the $\mathrm{HBC} \mathrm{h}_{1} \mathrm{PT}$ formulation), and despite the apparent large number of LECs, there is still a great deal of predictive power for specific processes.

\subsection{Loops, low energy counting and the HBChPT framework.}

As in the GB sector, unitarity and tadpole corrections are very important in the baryon sector. Their calculation involves loop diagrams, among them diagrams containing one or more baryon propagators, and thus the large mass scale $\stackrel{m}{m}$. This large scale has unwanted consequences for the low energy power counting of the loop integrals (assumed they are calculated in dimensional regularization when they are UV divergent). Indeed, the integrals in general show terms that are "fractional powers" of the heavy mass, i.e., contributions of the form $\stackrel{o}{m}^{n-\epsilon} / \epsilon$, that clestroy the hope of a low energy power counting for such loop diagrams. To illustrate this more explicitly, consider the calculation of the baryon self-energy (here given for the case of two flavors) that involves the diagrams depicted in Fig. 5. Calculated in the covariant framework with dimensional regularization the mass shift is $\left(g_{\mathrm{A}}\right.$ being the axial-vector coupling of the nucleon):

$$
\delta m=\stackrel{o}{m}^{3} \frac{3 g_{A}^{2}}{2(4 \pi)^{2} F_{0}^{2}}\left(\bar{c}_{0}+\bar{c}_{1} \frac{M_{\pi}^{2}}{\stackrel{o}{m}^{2}}-\pi \frac{M_{\pi}^{3}}{\stackrel{o}{m}^{3}}-\frac{1}{2} \frac{M_{\pi}^{4}}{\stackrel{o}{m}^{4}} \log \frac{M_{\pi}^{2}}{\stackrel{o}{m}^{2}}+\cdots\right) .
$$

This illustrates the problem mentioned before: the term proportional to $\bar{c}_{0}$ is of $\mathcal{O}\left(p^{0}\right)$. Thus, renormalization of the LO mass term was required. Although there is nothing wrong with this calculation, since after renormalization has been properly carried out the final results can be ordered consistently in the low energy expansion, it is more convenient to have a framework where such LO renormalizations are not required. This problem can be avoided because the terms that cause the difficulty are of short distance type, controlled by the baryon mass, and it is, therefore, possible to dispose of then right away by either the method of expanding in the inverse of the baryon mass to be described immediately, or by a convenient new regularization of the covariant theory as in CBChPT.

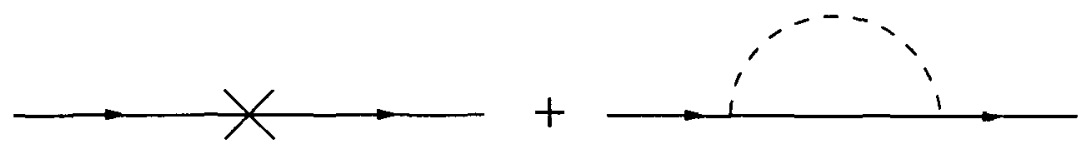

Fig. 5. Baryon self-energy. 
To illustrate the irlea of the $1 / m$ expansion, consider the scalar integral:

$$
\begin{aligned}
I & =\left.\int \frac{\mathrm{d}^{d} p}{(2 \pi)^{d}} \frac{\mathrm{i}}{p^{2}-M_{\pi}^{2}} \frac{\mathrm{i}}{(P-p)^{2}-\stackrel{o}{m}^{2}}\right|_{P^{2}=m^{2}} \\
& =-\frac{\mathrm{i}}{(4 \pi)^{2}}\left(\frac{1}{\epsilon}-\gamma_{\mathrm{E}}+2-\log \frac{\stackrel{o}{m}^{2}}{\mu^{2}}+\mathcal{O}\left(\frac{M_{\pi}}{\frac{o}{m}}\right)\right) .
\end{aligned}
$$

If $\stackrel{o}{m} \gg \Lambda_{\chi}$, one could expand the integrand in powers of $1 / \stackrel{o}{m}$, namely,

$$
\frac{1}{(P-p)^{2}-\stackrel{o}{m}^{2}}=\frac{-1}{2 P \cdot p}+\mathcal{O}\left(\frac{1}{\stackrel{o}{m}^{2}}\right) .
$$

At LO in this expansion the integrand does no longer contain the large mass, and one obtains:

$$
\bar{I}=\int \frac{\mathrm{d}^{d} p}{(2 \pi)^{d}} \frac{\mathrm{i}}{p^{2}-M_{\pi}^{2}} \frac{-\mathrm{i}}{2 P \cdot p}=-\frac{\mathrm{i}}{8 \pi} \frac{M_{\pi}}{\stackrel{o}{m}} .
$$

$\bar{I}$ has different UV behavior than $I$, which leads to the elimination from $\bar{I}$ of the terms explicitly displayed in $I$. This is however short distance physics that can be taken care of by renormalization. Thus, to order $1 / \stackrel{o}{m}, \bar{I}$ contains the same relevant low energy physics as $I$ to LO in $1 / \stackrel{o}{m}$. Clearly this expansion removes the possibility that the heavy mass enters through powers or logs: the so called fractional powers of the heavy mass mentioned above have been eliminated. How is the expansion implemented in the EFT? The idea is very simple and is equivalent to performing the Foldy-Wouthuysen transformation. The first thing is that in the limit $\stackrel{o}{m \rightarrow \infty}$ there is a superselection rule for the baryon's four-velocity $v_{\mu}$ for all low energy processes. One can then write:

$$
B(x)=\exp (-\mathrm{i} \stackrel{\circ}{m} v \cdot x)\left(B_{u^{1}}(x)+b_{v}(x)\right)
$$

where $\not p B_{v}=B_{v}$ and $\not b_{v}=-b_{v}$. For the propagation of a baryon. $B_{v}$, is the so called large component and $b_{v}$ the small one. As in the F-W transformation, the small component can be eliminated by use of the equations of motion it satisfies. In a path integral formulation it can be eliminated by integrating it out. The terms induced by eliminating the small component can then be expanded in powers of $1 / \stackrel{o}{m}$. Thus, by writing down the most general Lagrangian in terms of only the strong component one will be al)le to include those terms. The effective Lagrangian for the baryons will then have the general form: $\mathcal{L}_{\mathrm{HB}}=\bar{B}_{v} W B_{v}$, where the operator $W$ admits an expansion in $1 / \Lambda$, and $1 / m$ [40]. The Dirac structure of ${ }^{\circ}$ (an bo simplified using the Lo in $1 / \mathrm{im}$ relations [46]:

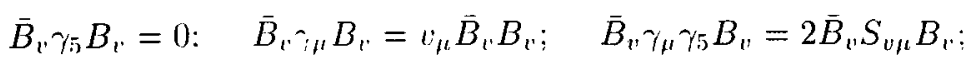

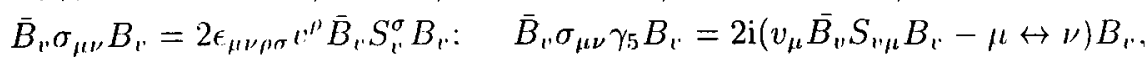


where $S_{1 \prime}^{\mu}$ is the spin operator satisfying the following relations:

$$
\begin{aligned}
& v \cdot S_{v}=0, \quad S_{v}^{2} B_{v}=-\frac{3}{4} B_{v^{\prime}} \\
& \left\{S_{l}^{\mu \prime}, S_{r}^{\prime \prime}\right\}=\frac{1}{2}\left(v^{\prime \prime} v^{\prime \prime}-g^{\prime \prime \prime}\right), \quad\left[S_{r^{\prime \prime}}^{\prime \prime}, S_{l^{\prime \prime}}^{\prime \prime}\right]=\mathrm{i} \epsilon^{\mu \nu \rho \sigma} v^{\prime \prime} S_{r}^{\sigma} .
\end{aligned}
$$

With this one can write down the most general Lagrangian in the $\mathrm{HBCl}_{2} \mathrm{PT}$ formulation. The lowest order Lagrangian, of $\mathcal{O}(p)$ is given by:

$$
\begin{aligned}
\mathcal{L}_{\mathrm{HB}}^{(1)}= & \left\langle\bar{B}_{v} \mathrm{i} v_{\mu} \nabla^{\mu} B_{u}\right\rangle \\
& +2 D\left\langle\bar{B}_{v} S_{v}^{\mu}\left\{\omega_{\mu}, B_{v}\right\}\right\rangle+2 F\left\langle\bar{B}_{v} S_{v}^{\mu}\left[\omega_{\mu}, B_{v}\right]\right\rangle .
\end{aligned}
$$

Terms in the Lorentz invariant Lagrangian $\mathcal{L}^{(1)}$ that are of order $1 / \stackrel{o}{m}$ move into $\mathcal{L}_{\mathrm{HB}}^{(2)}$. These terms required by Lorentz invariance implement the so called reparametrization invariance of the $\mathrm{HBChPT} \mathrm{[47],} \mathrm{an} \mathrm{example} \mathrm{of} \mathrm{which} \mathrm{is} \mathrm{the} \mathrm{ki-}$ netic term in $\mathcal{L}_{\mathrm{HB}}^{(2)}$ below, where a number of terms are displayed,

$$
\begin{aligned}
\mathcal{L}_{\mathrm{HB}}^{(2)}= & -\frac{1}{2 m}\left\langle\bar{B}_{v}, \nabla^{2} B_{v}\right\rangle+b_{\mathrm{D}}\left\langle\bar{B}_{v}\left\{\chi_{+}, B_{v}\right\}\right\rangle+b_{\mathrm{F}}\left\langle\bar{B}_{v}\left[\chi_{+}, B_{v}\right]\right\rangle \\
& +b_{0}\left\langle\bar{B}_{v} B_{v}\right\rangle\left\langle\chi_{+}\right\rangle+\mathrm{i} D\left\langle\bar{B}_{v}\left\{\{S \cdot \nabla, v \cdot \omega\}, B_{v}\right\}\right\rangle \\
& +\mathrm{i} F\left\langle\bar{B}_{v}\left[\{S \cdot \nabla, v \cdot \omega\}, B_{v}\right]\right\rangle+a_{1}\left\langle\bar{B}_{v} B_{v}\right\rangle\left\langle\omega^{2}\right\rangle \\
& +\left(\bar{B}_{v} B_{v}\right\rangle\left\langle(v \cdot \omega)^{2}\right\rangle+\cdots
\end{aligned}
$$

For two flavors, the effective Lagrangians of $\mathcal{O}\left(p^{3}\right)$ [48] and of $\mathcal{O}\left(p^{4}\right)$ [49] are also known.

The $1 / \stackrel{o}{m}$ expansion provides the baryon propagator at $\mathrm{LO}:-\mathrm{i} / p \cdot v$, that leads to loop integrals with the desired power counting. This power counting is easily derived, and for connected diagrams is given by:

$$
\nu_{\mathrm{B}}=4 L-2 I-I_{\mathrm{B}}+\sum_{i} \nu_{i} n_{i}=2+2 L+I_{B}+\sum_{i}\left(\nu_{i}-2\right) n_{i},
$$

where $L$ is the number of loops, $I$ the number of GB propagators and $I_{r m B}$ the number of baryon propagators. Note that this counting is valid only when a single baryon propagates through the diagram. If $\nu_{i}=1$ the vertex is a GB-baryon vertex. and since $I_{\mathrm{B}}=\sum_{i} n, \delta_{\nu_{i}-1}-1$. one immediately deduces that $\nu_{\mathrm{B}} \geq 2 L+1$. Let us now give a few illustrative applications of the $\mathrm{HBChPT}$.

\subsection{Baryon masses at order $p^{4}$}

The low energy expansion of the baryon self energy is rather straightfon warcl to calculate. It has the following expansion:

$$
m_{i}=\stackrel{i}{m}+\delta^{(2)} m_{i}+\delta^{(3)} m_{i}+\cdots
$$


where $\delta^{(n)} m$; contains quark masses to the power $n / 2$ (and chiral logs for $n$ even). At $\mathcal{O}\left(p^{2}\right)$, SU(3) breaking is linear in the quark masses, and therefore a GM-() relation holds:

$$
3 m_{\Lambda}+m_{\Sigma}-2\left(m_{\mathrm{N}}+m_{\Xi}\right)=0 .
$$

The deviation from this relation is of the order of $20 \mathrm{MeV}$, i.e., very small. At $\mathcal{O}\left(p^{3}\right)$ the first terms non-analytic in the quark masses appear, namely; $m_{q}^{3 / 2}[50]$. It turns out that these corrections are quite significant, indicating potential difficulties with the convergence of the expansion, in particular because of the large terms proportional to $M_{\mathrm{K}}^{3}$. It is on the other hand very important to notice that the GM-O relation receives a modest deviation of the order of $10 \mathrm{MeV}$ at this order [46]:

$$
\Delta_{\mathrm{GM}-\mathrm{O}}^{(3)}=\frac{M_{\mathrm{K}}^{3}}{2 \pi F_{0}^{2}}\left(1-\frac{2}{\sqrt{3}}\right)\left(\frac{D^{2}}{3}-F^{2}\right)+\mathcal{O}\left(M_{\pi}^{3}\right) .
$$

The inclusion of the $\mathcal{O}\left(p^{4}\right)$ contributions brings in LECs that are not directly known from phenomenology, requiring some modeling. An analysis to this order [51] gives the following numerical pattern of corrections up to $\mathcal{O}\left(p^{4}\right)$ :

$$
\begin{array}{ll}
m_{\mathrm{N}}=\stackrel{o}{m}(1+0.34-0.35+0.24), & m_{\Lambda}=\stackrel{o}{m}(1+0.69-0.77+0.54), \\
m_{\Sigma}=\stackrel{o}{m}(1+0.81-0.70+0.44), & m_{\Xi}=\stackrel{o}{m}(1+1.10-1.16+0.78),
\end{array}
$$

which shows an slow convergence rate of the expansion, in particular in the hyperon sector.

The analysis of the masses cannot be carried out exhaustively without considering the $\pi-\mathrm{N} \sigma$-term, which is defined by:

$$
\sigma_{\pi \mathrm{N}}(0)=\hat{m} \frac{\partial m_{\mathrm{N}}}{\partial \hat{m}}
$$

The reason is that both $\stackrel{o}{m}$ and the term proportional to $b_{0}$ give singlet contributions. and knowledge of the $\sigma$-term permits to separate them. This can be extracted by studying $\pi-\mathrm{N}$ scattering, in particular the appropriately subtracted D-amplitude at the so called Cheng-Dashen point [52]. This is a very important topic that also allows one to set bounds on the contribution to the nucleon mass by the strange quark mass.

Before closing this section let us go back to the issue of convergence. Is the pattern in (61) indicating a lack of convergence?. Naively it seems so, but a more sensible question is the following: does the global fit to data improve by going to higher orders at a rate that indicates convergence? This issue was recently discussed $[42,53]$, and a scheme was proposed to fit the LEC's order by order. in such a way that a reordering of the expansion results. The reordering is defined as follows: lot a LEC be expressed as

$$
L_{n}=L_{n}^{(0)}+L_{n}^{(1)}+L_{n}^{(2)}+\cdots
$$


where $L_{n}^{(0)}$ is the best fit at the order the LEC first contributes, etc. Consider now the expansion of an observable in the usual manner:

$$
A_{i}=A_{i}^{(0)}\left(\left\{L_{n}\right\}\right)+A_{f}^{(1)}\left(\left\{L_{n}\right\}\right)+A_{i}^{(2)}\left(\left\{L_{n}\right\}\right)+\cdots .
$$

One can instead reorder the series as follows:

$$
\left.A_{\ell}=A_{\ell}^{(0)}\left(\left\{L_{n}^{(0)}\right\}\right)+\left[A_{\ell}^{(1)}\left(\left\{L_{n}^{(0)}\right\}\right)+\sum_{n} \frac{\partial A_{\ell}^{(0)}}{\partial L_{n}}\left(\left\{L_{n}^{(0)}\right\}\right) L_{n}^{(1)}\right)\right]+\cdots
$$

Now instead of taking as the LO result $A_{\ell}^{(0)}\left(\left\{L_{n}\right\}\right)$, one takes $A_{\ell}^{(0)}\left(\left\{L_{n}^{(0)}\right\}\right)$, etc. Clearly this procedure improves the convergence, since it systematically tends to minimize the size of the NLO corrections. An explicit analysis of the masses [53] shows a dramatic improvement of the situation when applying the reordering:

$$
\begin{aligned}
& m_{\mathrm{N}}=\bar{m}\left(1-0.18+0+2.6 \times 10^{-3}\right), \quad m_{\Lambda}=\bar{m}\left(1-0.034+10^{-3}+6.9 \times 10^{-3}\right), \\
& m_{\Sigma}=\bar{m}(1+0.034+0+0), \quad m_{\Xi}=\bar{m}(1+0.148+0+0),
\end{aligned}
$$

where the best fit mass $\bar{m}$ is equal to $1150 \mathrm{MeV}$. Although it is still to be shown in a systematic way that this kind of reordering works for other observables, this result is certainly very encouraging.

\subsection{The Goldberger-Treiman relation}

One of the fundamental relations implied by chiral symmetry in the baryon sector is the Goldberger-Treiman relation [54]. It holds in the chiral limit and it is an identity derived from $\mathcal{L}_{\mathrm{B}}^{(1)}$ when the equations of motion are imposed, and reads:

$$
\stackrel{o}{m} g_{\mathrm{A}}^{a b c}=F_{0} g_{c a b}, \quad a=1, \cdots, 8, \text { etc, }
$$

where $g_{A}^{a b c}$ is the coupling of the axial-vector current $A_{\mu k}^{c}$ between the baryon states $a$ and $b$, and $g_{c a b}$ is the coupling of the GB $c$ to the same baryon states. In particular for the nucleons one can write:

$$
m_{\mathrm{N}} g_{\mathrm{A}}=F_{\pi} g_{\pi \mathrm{NN}}
$$

which indeed holds at the few percent level. The explicit breaking of chiral symmetry by the quark masses produces a deviation from the GTR. The relation can instead be written in the form:

$$
\frac{m_{a}+m_{b}}{2} g_{A}^{a b c}\left(q^{2}=0\right)=\frac{1-\Delta^{a b c}}{M_{c}^{2}} g_{c a b}\left(q^{2}=M_{c}^{2}\right) P^{c}\left(q^{2}=M_{c}^{2}\right) .
$$

where $P^{c}$ is the pseudoscalar decay constant of the GB $c$, and $\lambda^{\text {alc }}$ is the so called Goldberger-Treiman discrepancy (GTD). Using the exact result:

$$
\frac{m_{a}+m_{b}}{2} g_{A}^{a b r}\left(q^{2}=0\right)=\frac{1}{M_{c}^{2}} g_{c a b}\left(q^{2}=0\right) P^{c}\left(q^{2}=0\right)
$$


the GTD can be written as:

$$
\Delta^{a b c}=\frac{N^{a b c}\left(M_{c}^{2}\right)-N^{a b c}(0)}{N^{a b c}\left(M_{c}^{2}\right)}
$$

with

$$
N^{a b c}\left(q^{2}\right)=g_{c a b}\left(q^{2}\right) P^{c}\left(q^{2}\right)+\left(q^{2}-M_{c}^{2}\right) \delta^{a b c} .
$$

The LO contributions to the GTD are as expected of $\mathcal{O}\left(p^{2}\right)$, and stem from terms in the $\mathcal{O}\left(p^{3}\right)$ Lagrangian (given here in the isospin limit):

$$
\mathcal{L}_{\mathrm{GTD}}^{(3)}=-\mathrm{i} F_{19}\left\langle\bar{B}_{u} S_{v}^{\mu}\left[\nabla_{\mu} \chi_{-}, B_{v}\right]\right\rangle-\mathrm{i} D_{19}\left\langle\bar{B}_{v} S_{v}^{\mu}\left\{\nabla_{\mu} \chi_{-}, B_{v^{\prime}}\right\}\right\rangle,
$$

which gives: $\Delta^{a b c}=\delta^{a b c} /\left(g_{\mathrm{A}}^{a b c} \stackrel{o}{m}\right)$, with [55]

$$
\begin{aligned}
\delta^{a b c}= & 4 \stackrel{\circ}{m} B_{0}\left(2 \mu^{0}\left(\mathrm{i} F_{19} f^{a b c}+D_{19} d^{a b c}\right)\right. \\
& \left.+d^{c d e} \mu^{d}\left(\mathrm{i} F_{19} f^{b e a}+D_{19} d^{a b e}\right)+\frac{2}{3} \mu^{c} D_{19} \delta_{a b}\right),
\end{aligned}
$$

with: $\mu^{0}=\frac{1}{3}\left(2 \hat{m}+m_{s}\right)$ and $\mu^{a}=-\frac{2}{\sqrt{3}} \delta_{a 8}\left(m_{s}-\hat{m}\right)$. One can show that at this order the so called Dashen-Weinstein relation holds exactly (as for GM-O, it is due to symmetry breaking by an octet operator):

$$
M_{\mathrm{K}}^{2}\left(\frac{g_{\mathrm{A}}}{g_{\mathrm{V}}}\right)^{\mathrm{NN \pi}} \Delta^{\mathrm{NN} \pi}=\frac{1}{2} M_{\pi}^{2}\left(3\left(\frac{g_{\mathrm{A}}}{g_{\mathrm{V}}}\right)^{\mathrm{N} \Lambda \mathrm{K}} \Delta^{\mathrm{N} \Lambda \mathrm{K}}-\left(\frac{g_{\mathrm{A}}}{g_{\mathrm{V}}}\right)^{\mathrm{N} \Sigma \mathrm{K}} \Delta^{\mathrm{N} \Sigma \mathrm{K}}\right) .
$$

The very interesting aspect of the GTR is that the corrections to the GTDs are of $\mathcal{O}\left(p^{4}\right)$ [55]. The absence of corrections of $\mathcal{O}\left(p^{3}\right)$ can be checked by explicit calculation of the one-loop corrections to the quantity $N^{a b c}\left(q^{2}\right)$ defined above; the $\mathcal{L}_{\mathrm{HB}}^{(4)}$ gives no contributions either. Since for all other quantities involved in the D-W relation the corrections are two orders higher in the power counting, that relation also gets corrected in the same fashion. This makes the DW relation into a reliable tool to relate the discrepancies. In particular, one can use it to determine what, a reasonable size for $\Delta^{N N \pi}$ is. With the well known values for the ratios $g_{A} / g_{V}$, one. can immediately infer that if the other two discrepancies are in the $50 \%$ range, then $\left|\Delta^{\mathrm{NN} \pi}\right|<3 \%$ or so. Even more, one can use results for $g_{\mathrm{KN} A}$ and $g_{\mathrm{K}} \mathrm{x}$ ob trained in a not so well established analysis [56]. and a best fit to the resulting cliscrepancies give $\Delta^{\mathrm{N} \pi}=0.018 \pm 0.005[55]$ and $g_{\pi \mathrm{NN}}=13.14 \pm 0.1$. A group of analyes [57] is consistent with this. while other [58] obtains values that seem too large to be consistent with the GTD analysis.

\section{$9 \quad$ More applications}

HBChPT has been applied to a large number of processes: $\pi-\mathrm{N}$ scattering, pion photo- and electro-production near threshold, Compton scattering, weak decays of 
hyperons, etc. A great doal of new understanding has been achieved. and at tho same time there is still much more to do and improve. At the same time. several experimental programs have provided precision data that has been very inportant. as is the case with photo- and clectro-production.

In this section I briefly focus on a few very important results in photo-production and in Compton scattering.

\subsection{Photo-production}

The photo-production process $\gamma \mathrm{N} \rightarrow \pi \mathrm{N}^{\prime}$ has received close attention as a process for testing the ChPT predictions. Since the realization that the higher order corrections are very important in the $\pi^{0}$ case [59], this particular process has been studied in much detail, both theoretically as well as experimentally. Cnlike the charged pion case, where the amplitude is dominated by the contact term imposer by EM gauge invariance, the Kroll-Ruderman term (that derives from the terms proportional to $\omega_{\mu}$ in $\mathcal{L}^{(1)}$ with the EM field as source), the $\pi^{0}$ process proceeds first at higher order in the chiral expansion.

Near threshold the amplitude expanded in multipoles has the form:

$$
A_{\gamma-\operatorname{prod}}=\frac{4 \pi \sqrt{s}}{m_{N}}\left(\vec{\sigma} \cdot \vec{\epsilon}\left(E_{0+}+\hat{q} \cdot \hat{k} P_{1}\right)+\cdots\right)
$$

where $k$ and $\vec{\epsilon}$ are respectively the photon momentum and polarization, $\vec{\sigma}$ the nucleon spin operator, and $q$ the momentum transfer to the nucleon. The S-wave electric dipole amplitude $E_{0+}$ has been studied in much detail. For charged pions the K-R term gives the LO contribution at threshold:

$$
E_{0+}\left(\pi^{+} n\right)=\frac{\left(g_{\mathrm{A}}\right.}{4 \pi \sqrt{2}\left(1+\frac{M_{\pi}}{m_{N}}\right)^{3 / 2}}, \quad E_{0+}\left(\pi^{-} p\right)=-\frac{e g_{\mathrm{A}}}{4 \pi \sqrt{2}\left(1+\frac{M_{\pi}}{m_{\mathrm{N}}}\right)^{1 / 2}} .
$$

Both turn out to be in good agreement with the experimentally extracted ones [60]. On the other hand, for the $\pi^{0}$, the electric dipole amplitudes are of $\mathcal{O}(p)$ in the case of $\pi^{0} p$ and of $\mathcal{O}\left(p^{2}\right)$ in the case of $\pi^{0} n$, and at threshold are given by:

$$
\begin{aligned}
& E_{0+}\left(\pi^{0} p\right)=-\frac{e g_{A}}{8 \pi F_{\pi}}\left\{\left[\frac{M_{\pi}}{m_{\mathrm{N}}}-\frac{M_{\pi}^{2}}{2 m_{\mathrm{N}}^{2}}\left(3+\kappa_{p}\right)\right]-\frac{M_{\pi}^{2}}{\left(4 F_{\pi}\right)^{2}}\right\}+\mathcal{O}\left(p^{3}\right), \\
& E_{0+}\left(\pi^{0} n\right)=-\frac{e g_{\mathrm{A}}}{8 \pi F_{\pi}}\left[\left(\frac{M_{\pi}^{2}}{2 m_{\mathrm{N}}^{2}} \kappa_{n}\right)-\frac{M_{\pi}^{2}}{\left(4 F_{\pi}\right)^{2}}\right]+\mathcal{O}\left(p^{3}\right) .
\end{aligned}
$$

The terms proportional to $1 / F_{\pi}^{3}$ originate in one loop diagrans. and leepresent a very large correction to the terms in the curly brackets [59]. This implies the need of including higher order terms in order to have some predictivity. Such calculations have been carried out to $\mathcal{O}\left(p^{3}\right)[61]$ and even to $\mathcal{O}\left(p^{4}\right)$ [62], where reasomably good agreement with the data [63] is achieved:

$$
\begin{aligned}
E_{0+}||_{\mathrm{HBChPT}}\left(\pi^{0} p\right) & =-1.16 \times 10^{-3} M_{\pi}^{-1}, \\
E_{0+} \mid \operatorname{Exp}_{\mathrm{p}}\left(\pi^{0} p\right) & =(-1.32 \pm 0.07) \times 10^{-3} M_{\pi}^{-1} .
\end{aligned}
$$


The level of predictivity of ChPT in this process is reduced by the need to go to higher orders, but this mostly affects the values of the $E_{0+}\left(\pi^{0} N\right)$ at threshold. while the energy clependence is well described at the one-loop level [61.62].

$\mathrm{P}$-wave multipoles are, on the other hand, very successfully described by ChPT [60]. The extension to electro-production has also been worked out as well [64]. and some interesting observations have been drawn from the existing data. that is above $Q^{2}=0.1 \mathrm{GeV}^{2}$, and which is estimated to be a bit high for the applicalsility of ChPT.

\subsection{Compton scattering}

Obviously, among the most accessible properties of baryons are their EM ones. Of particular interest to the low energy expansion is Compton scattering on nucleons. There are some famous low energy theorems: at LO the Compton amplitude is given by the Thompson term that only depends on the mass and charge of the particle, and at NLO $\left(\mathcal{O}\left(\omega_{\gamma}\right)\right.$ in the Lab frame) the Gell-Mann-Low theorem states that the amplitude is given in terms of the anomalous magnetic moment of the particle. At NNLO $\left(\mathcal{O}\left(\omega_{\gamma}^{2}\right)\right)$ the electric and magnetic Compton polarizabilities enter. These quantities turn out to be very sensitive to the pion cloud of the nucleons, and thus to a great extent they can be described within ChPT. These contributions are proportional to $1 / M_{\pi}$, indicating that in the chiral limit ChPT ought to give a perfect description. At LO the electric polarizabilities are given by [65]:

$$
\bar{\alpha}_{p}=\bar{\alpha}_{n}=\frac{5 \alpha_{\mathrm{EM}} g_{\mathrm{A}}^{2}}{96 \pi F_{\pi}^{2} M_{\pi}} \simeq 12 \times 10^{-4} \mathrm{fm}^{3}
$$

This result agrees remarkably well with the experimental one for the proton [66]: $(12.1 \pm 0.8 \pm 0.5) \times 10^{-4} \mathrm{fm}^{3}$. For the neutron one expects that a similar agreement will result when $\bar{\alpha}_{n}$ becomes better determined experimentally.

The magnetic polarizabilities are trickier. The reason is that one expects a large paramagnetic contribution due to the $M_{1}$ transition to the $\Delta$ resonance. This is numerically a large effect, much larger than the values of the polarizabilities themselves. In the chiral counting the LO contribution is due to the pion clond (term proportional to $M_{\pi}^{-1}$ ). and the $\Delta$ contribution is of NLO. However, the former is smaller than the latter. This indicates the need to look at the NNLO (ontributions, which was done sometime ago [67]. Indeed, it is observed that the NNLO contributions are dominantly diamagnetic and tend to cancel the $\Delta$ contribution. Unfortunately, due to the large cancelation required, the predictivity of ChPT for the magnetic polarizabilities is very limited.

Finally, there are higher order polarizabilities [68] associated with higher order terms in the Compton amplitude expansion, as well as generalized polarizabilities that enter in virtual Compton scattering [69]. 


\section{Manifestly covariant EFT}

The $1 / m$ expansion employed to define $\mathrm{HBChPT}$, while very useful in practice. it is not free of inconsistencies. Low energy effects that are non-analytic in $1 / m$ are necessarily lost. Among such effects are the anomalous thresholds that appear in diagrams such as the one shown in Fig. 6. The explicit calculation of this diagram with relativistic scalar particle propagators gives:

$$
\gamma(t)=\gamma_{0}(t)+\delta \gamma(t)
$$

where $t=q^{2}$. The first term reads:

$$
\begin{aligned}
\gamma_{0}(t)= & \frac{1}{32 \pi m M_{\pi}}\left(\frac{1}{\sqrt{\tau}} \log \frac{2+\sqrt{\tau}}{2-\sqrt{\tau}}\right. \\
& \left.+\frac{2 M_{\pi}}{m} \frac{2-\tau}{\pi \sqrt{\tau(4-\tau)}} \arcsin \frac{\sqrt{\tau}}{2}+\frac{M_{\pi}}{2 m}\left(\log \frac{M_{\pi}}{m}-1\right)\right),
\end{aligned}
$$

where $\tau \equiv t / M_{\pi}^{2}$. This first term shows the normal threshold at $t=4 M_{\pi}^{2}$, and its relevant low energy physics can be obtained in the $1 / m$ expansion. On the other hand, the second term is given by:

$$
\delta \gamma(t)=\frac{1}{32 \pi m M_{\pi}}\left(\frac{M_{\pi}}{m \sqrt{4-\tau}}-\log \left(1+\frac{M_{\pi}}{m \sqrt{4-\tau}}\right)\right) .
$$

It is easy to check that $\delta \gamma(t)$ has a cut (that belongs in the second Riemann sheet) for $t$ below the anomalous threshold $4 M_{\pi}^{2}-M_{\pi}^{4} / \mathrm{m}^{2}$. This feature is lost in the $1 / m$. expansion. Recently, for the propagation of a single heavy particle, a framework that corrects the deficiencies of the $1 / \mathrm{m}$ expansion and has all the desired properties for the EFT was found by Ellis and Tang [41] and further developed and elegantly formulated by Becher and Leutwyler [42]. It is based on a manifestly Lorentz covariant formulation with a cleverly implemented regularization that eliminates the high energy poles of the heavy particle propagators, allowing in this way to retain the same low energy power counting valid in the $1 / m$ expansion. To illustrate the

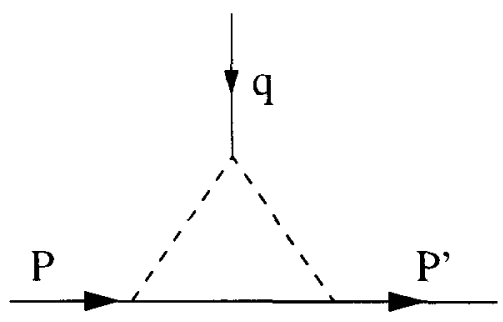

Fig. 6. Loop contribution and counterterm to the nucleon mass. 
method it is convenient to consider the second self energy diagram in Fig. 5 for scalar particles and in dimensional regularization. The loop integral is:

$$
I=-\mathrm{i} \frac{\Gamma(\epsilon)}{(4 \pi)^{2-\epsilon}} \int_{0}^{1} \mathrm{~d} x \frac{1}{\left(x m^{2}+(1-x) M_{\pi}^{2}-x(1-x) P^{2}\right)^{\prime}} .
$$

where $P^{2}=m^{2}+\mathcal{O}(m p)$ with $p$ the generic small energy scale. The zeroes of the denominator are located within a radius of the order of $p / m$ from $x=0$. This means that the domain of $x$ near the origin gives the IR contributions to the integral, while the domain near $x=1$ gives short distance contributions. The decomposition

$$
\int_{0}^{1} \cdots=\int_{0}^{\infty} \cdots-\int_{1}^{\infty} \cdots
$$

suggests itself. Indeed. the so called regular part $I_{\mathrm{R}}$ resulting from the domain of integration $[1, \infty)$ is analytic in the whole low energy region (measured here by the virtuality of $P$ ), and consists only of terms that are fractional powers [42] of $\mathrm{m}$ that lead to the $\log m^{2}$ term as $d \rightarrow 4$. In the low energy region the contributions from $I_{\mathrm{R}}$ are equivalent to contributions from local counter-terms. On the other hand, the integral over the domain $[0, \infty)$, called the irregular or low energy part and clenoted by $\bar{I}$, is the one that retains the low energy analytic structure of the diagram. The important point is that $\bar{I}$ is free of pieces that are fractional powers of $m$, as one can see from its explicit expression:

$$
\begin{aligned}
\bar{I}= & -\frac{\mathrm{i}}{32 \pi^{2}}\left\{\frac{P^{2}-m^{2}+M \pi^{2}}{P^{2}}\left(\frac{1}{\epsilon}-\gamma_{\mathrm{E}}+\log 4 \pi-\log M_{\pi}^{2}\right)\right. \\
& \left.-\frac{1}{P^{2}} \sqrt{-\lambda\left(P^{2}, m^{2}, M_{\pi}^{2}\right)}\left[\pi+2 \arctan \left(\frac{P^{2}-m^{2}+M_{\pi}^{2}}{\sqrt{-\lambda\left(P^{2}, m^{2}, M_{\pi}^{2}\right)}}\right)\right]\right\},
\end{aligned}
$$

with $\lambda$ the usual phase space function.

Given that in the low energy domain $I_{\mathrm{R}}$ can be considered as a local contribution, one can dispose of it without any harm, as genuine contributions that are lost in doing this can be recovered by adding the appropriate counter-terms to the Lagrangian. Thus, the regulated self energy integral can be identified with $\bar{I}$ instead of $I$. This defines a new regularization, that has been named " infrared regularization" in [42]. The regularization so defined preserves the symmetries of the theory in the same way as dimensional regularization does [42]. The extension of the IR regularization to other one-loop diagrams can be carried out using the following simple rules $[42]$ :

i) Combine the light particle propagators with one set of Feynman parameters. and the heary ones with another set.

ii) Combine the results of applying (i) with a Feymman parameter whose integration domain is then extended in a similar fashion as for the self-energy diagran.

One point to be noted here is that the UV divergences contain in general higher order terms in the low energy expansion, as it can be seen from the self-energy. 
diagram if one expands $1 / P^{2}$ around $P^{2}=m^{2}$. This requires an infinite string of counterterms in the Lagrangian to elininate the UV divergence. The problem is however easily solved: if one is working at a given order in the low energy expansion. then one should neglect the higher order terms in the UV divergent piece of the loop integral, and thus (arry ont the renormalization consistently up to that order.

The baryon ChPT defined with the IR regularization method is called corariant BChPT, or in short, CBChPT. So far a few applications of CBChPT hare been worked out. The baryon masses have been considered $[42,70]$, and also the scalar form factor of the nucleon ( $\sigma$-term) [42]. Other applications will follow, and it is to be expected that ther will have a substantial impact on the current status of baryon ChPT.

Finally, in the sector of more than one baryon, as is the case of the NN system, the $1 / m$ expansion cannot be applied at all as it misses the two-heavy-particle cut. In this case an extension of the IR regularization can be worked out as it was shown recently [71].

\section{Spin 3/2 baryons: in or out?}

In the large $N_{c}$ limit, the low lying baryons belong into a spin-flavor multiplet where the states with small values of the isospin are nearly degenerate (splittings of $\mathcal{O}\left(I / N_{c}\right)$ ). In that limit the effective theory would not only have to include explicitly the spin $1 / 2$ baryons, but also states of higher spin as well. Translated to $N_{\mathrm{c}}=3$, this indicates that the inclusion of the spin $3 / 2$ baryons should be considered seriously. Its proximity in mass to the low lying spin $1 / 2$ baryons implies that the low energy expansion can be improved considerably by including them explicitly. The only reason one is justified to include them is that in a certain limit, these resonances are narrow, and nearly stable states can be dealt with consistently in the EFT. This was emphasized in the works of Jenkins and Manohar [40, 46]. where they showed that the inclusion of the spin $3 / 2$ decouplet $\left(\Delta, \Sigma^{*}\right.$, etc) brings in cancelations in loop diagrams that indeed improve the behavior of the low energy. expansion. The interesting thing is that these cancelations originate in relations that are required for the large $N_{c}$ expansion to be consistent $[72-74]$. Since I have already used all the space available, I will not discuss any details about the technical issues involved in including the spin $3 / 2$ baryons in HBChPT, and rather refer to recent works $[75]$.

I am very thankful to the organizers of the Indian Summer School for the excellent progran they put together and for their wonderful hospitality that made for a delightful visit to Prague. I would like to thank in particular. Jiř Adan and Jiři Hošek for the kind invitation to present these lectures. and Carlos Schat for a critical reading of the manuseript. This work is partially supported by the Department of Energy through contract DE-ACo:84ER40150 (JLG). by the National Science Foundation through grant \# PHY-9733343. and by sabbatical leave support from the Southeastern Universities Research Association. 


\section{References}

[1] S. Weinberg: Physica A 96 (1979) 327.

[2] J. Gasser and H. Lentwyler: Ann. of Phys. 158 (1984) 142.

[3] J. Gasser and H. Leutwyler: Nincl. Phys. B 250 (1985) 465.

[4] G. Ecker: Chiral Symmetry, hep-ph/9805500.

A. Pich: Effective Field Theory, hep-ph/9806303.

U.-G. Meißner: Chiral Nucleon Dynamics, in Themes in Strong Interactions

(Ed. J.L. Goity), World Scientific, Singapore, 1998, p. 139.

B.R. Holstein: Introduction to Chiral Perturbation Theory, in Hadronic structure (Ed. J.L. Goity), World Scientific, Singapore, 1998, p. 58.

[5] B. Holstein: Am. J. Phys. 67 (1999) 422.

[6] H. Euler: Ann. Phys. 26 (1936) 398.

H. Euler and W. Heisenberg: Z. Phys. 98 (1936) 714.

[7] J.D. Bjorken and S.D. Drell: Relativistic Quantum Mechanics, McGraw-Hill, New York, 1964.

[8] G. 'tHooft: in Recent Developments in Gauge Theories (Eds. G. 'tHooft et al.), Plenum Press, 1980.

[9] T. Banks and A. Casher: Nucl. Phys. B 169 (1980) 103.

E. Marinari, G. Parisi and C. Rebbi: Phys. Rev. Lett. 47 (1981) 1795.

[10] C. Vafa and E. Witten: Phys. Rev. Lett. 53 (1984) 535.

[11] J. Goldstone: Nuovo Cim. 19 (1961) 154.

Y. Nambu: Phys. Rev. Lett. 4 (1960) 380.

[12] S. Coleman, J. Wess and B. Zumino: Phys. Rev. 177 (1969) 2239.

C. Callan, S. Coleman, J. Wess and B. Zumino: Phys. Rev. 177 (1969) 2247.

[13] S. Weinberg: Phys. Rev. Lett. 16 (1966) 879, ibid 17 (1966) 616.

[14] G. Colangelo: Theory of $\pi-\pi$ scattering, to appear in proc. of Chiral Dynamics: theory and experiment III. (Eds. A. Bernstein, J.L. Goity, and U.-G. Meißner), World Scientific, in press.

[15] D.B. Kaplan and A.V. Manohar: Phys. Rev. Lett 56 (1986) 2004.

[16] R. Kaiser and H. Leutwyler: Eur. Phys. J. C 17 (2000) 623.

[17] H. Leutwyler: Phys. Lett. B 378 (1996) 313, and hep-ph/9609467.

[18] G. Ecker, J. Gasser. A. Pich and E. de Rafael: Nucl. Phys. B 321 (1989) 311.

G. Ecker, J. Gasser. H. Leutwyler, A. Pich and E. de Rafael: Phys. Lett. B 223 (1989) 425 .

[19] J. Gasser and H. Lentwyler: Nucl. Phys. B 250 (1985) 517.

[20] S.M. Roy: Phys. Lett. B 36 (1971) 353.

[21] B. Ananthanarayan. G. Colangelo, J. Gasser and H. Leutwyler: hep-ph/0005297.

[22] G. Colangelo, J. Gasser and H. Leutwyler: hep-ph/0007112.

[23] G. Amoros, J. Bijnens and P. Talavera: Nucl. Phys. B 585 (2000) 293. 
[24] M. Zeller: A new monsurement of the $\mathrm{K}_{e, 4}^{+}$decay, to appear in proc. of Chiral Dynam. ics: theory and experiment III. (Eds. A. Bernstein, J.L. Goity, and U.-G. Meißner), World Scientific. in press.

[25] J. Schacher: Expcrimental status of pionium at $C E R N$, to appear in proc. of Chiral Dynamics: theory and experiment III. (Eds. A. Bernstein, J.L. Goity; and U.G. Meißner), World Scientific, in press.

[26] S.L. Adler: Phys. Rev. 177 (1969) 2426.

J.S. Bell and R. Jackiw: Nuovo. Cim. 60A (1969) 47.

[27] K. Fujikawa: Phys. Rev. D 21 (1980) 2848.

[28] J. Wess and B. Zumino: Phys. Lett. B 37 (1971) 95.

[29] E. Witten: Nucl. Phys. B 223 (1983) 422.

[30] A. Gasparian et al.: A precision measurement of the $\pi^{0}$ lifetime via the Primakoff effect, JLab experiment E-99-014.

[31] B. Moussallam: Phys. Rev. D 51 (1994) 4939.

[32] A. Bernstein, J.F. Donoghue, J.L. Goity and B. Holstein: in preparation.

[33] J. Bijnens, A. Bramon and F. Cornet: Phys. Rev. Lett. 61 (1988) 1453.

[34] J.F. Donoghue and B.R. Holstein: Phys. Rev. D 40 (1989) 2378.

B.R. Holstein: Comments Nucl. Part. Phys. 19 (1990) 239.

[35] F. Gilman and M. Wise: Phys. Rev. D 21 (1980) 3150.

[36] J. Kambor, J. Missimer and D. Wyler: Nucl. Phys. B 346 (1990) 17

[37] G. D'Ambrosio and D. Espriú: Phys. Lett. B 175 91986) 237.

J.L. Goity: Z. Phys. C 34 (1987) 341.

[38] G. Isidori: Weak decays of pseudo-Goldstone bosons, to appear in proc. of Chiral Dynamics: theory and experiment III.

(Eds. A. Bernstein, J.L. Goity, and U.-G. Meißner) World Scientific, in press.

[39] J.F. Donoghue, J. Gasser and H. Leutwyler: Nucl. Phys. B 343 (1990) 341.

J.F. Donoghue: Dispersion relations in effective field theory, hep-ph/9607351 and references therein.

[40] E. Jenkins and A.V. Manohar: Phys. Lett. B 255 (1991) 558.

[41] H-B. Tang: hej-ph/9607436.

P.J. Ellis and H-B. Tang: Phys. Rev. C 57 (1998) 3356.

[42] Th. Becher and H. Leutwyler: Eur. Phys. J. C 9 (1999) 643.

[43] See for instance U.-G. Meißner: "Chiral QCD: Baryon Dynamics", in Festschrift in honor of Boris Ioffe. hep-ph/0007092.

[44] J. Gasser, M.E. Sainio and A. Šrarc: Nucl. Phys. B 307 (1988) 779.

[45] A. Krause: Helv. Plịs. Acta 63 (1090) 3.

[46] E. Jenkins and A.V. Manohar: in Effective Field Theories of the Standard Model (Ed. U.-G. Meißner), World Scientific, Singapore, 1992. p. 113.

[47] M. Luke and A. N. Manohau: Phys. Lett. B 286 (1992) 348.

[48] G. Ecker: Phrs. Lett. B 336 (1994) 508.

G. Ecker and M. Mojżis: Phys. Lett. B 365 (1996) 312. 
[49] N. Fettes, L.-G. Mreibner, M. Mojžiš and S. Steininger: Ann. of Plyss. 283 (2000) 273.

[50] J. Gasser and A. Zepeda: Nucl. Phys. B 174 (1980) 445.

[51] B. Borasoy and U.-G. Meißnex: Ann. of Phys. 254 (1997) 192.

[52] J. Gasser, H. Leutwrler and M.E. Sainio: Phys. Lett. B 253 (1991) 252. and reference's therein.

[53] M. Mojžiš and J. Kambor: Phys. Lett. B 476 (2000) 344.

[54] M.L. Goldberger and S.B. Treiman: Phys. Rev, 110 (1958) 1178.

[55] J.L. Goity, R. Lewis, M. Schvellinger and L. Zhang: Phys. Lett. B 454 (1999) 115.

[56] R.G.E. Timmermans, T.A. Rijken and J.J. de Swart: Nucl. Phys. A 585 (1995) 143c.

[57] V. Stoks, R. Timmermans and J.J. de Swart: Phys. Fev. C 47 (1993) 512, and $\pi$ N Newslett. 13 (1997) 96.

R.A. Arndt, I.I. Strakovsky and R.L. Workman: Phys. Rev. C 50 (1994) 2731.

R.A. Arndt et al.: Phys. Rev. C 49 (1994) 2729; Phys. Rev. Lett. 68 (1992) 1653.

M.M. Pavan and R.A. Arndt: $\pi$ N Newslett. 15 (1999) 171.

[58] J. Rahm et al.: Phys. Rev. C 57 (1998) 1077.

R. Koch and E. Pietarinen: Nucl. Phys. A 336 (1980) 331.

T.E.O. Ericson, B. Loiseau and A.W. Thomas: hep-ph/0009312.

[59] V. Bernard, N. Kaiser, J. Gasser and U.-G. Meißner: Phys. Lett. B 268 (1991) 291.

[60] V. Bernard, N. Kaiser and U.-G. Meissner: Phys. Lett. B 393 (1996) 116.

[61] V. Bernard, N. Kaiser and U.-G. Meissner: Z. Phys. C 70 (1996) 483.

[62] V. Bernard, N. Kaiser and U.-G. Meissner: Phys. Lett. B 378 (1996) 337.

[63] P. Argan et al.: Phys, Lett. B 206 (1988) 4.

J.C. Bergstrom et al.: Phys. Rev. C 53 (1996) R1052.

[64] V. Bernard, T-S.H. Lee, N. Kaiser and U.-G. Meissner: Phys. Rep. 246 (1994) 315.

V. Bernard, N. Kaiser and U.-G. Meissner: Nucl. Phys. A 607 91996) 379.

[65] V. Bernard, N. Kaiser and U.-G. Meißner: Phys. Rev. Lett. 67 (1991) 1515

[66] F.J. Federspiel et al: Plyys. Rev. Lett. 67 (1991) 1511.

A. Zeiger et al.: Phys. Lett. B 278 (1992) 34.

E. Hallin et al.: Phys. Rev. C 48 (1993) 1497.

B. MacGibbon et al.: Physs. Rev. C 52 (1995) 2097.

[67] V. Bernard, N. Kaiser, A. Schmidt and U.-G. Meißner: Phys. Lett. B 319 (1993) 269.

[68] B.R. Holstein: "Nucleon polarizabilities", and references therein, to appear in proc. of Chiral Dynamics: theory and experiment III. (Eds. A. Bernstein. J.L. Goity: and U.-G. Meißner), World Scientific, in press.

[69] Th. Hemmert: Nucleon Compton scattering in chiral effective theorics, and references therein, to appear in proc. of Chiral Dynamis:s: theory and experimcnt. III. (Eds.

A. Bernstein, J.L. Goitr: and L.-G. Meißner), World Scientific: in press.

[70] P.J. Ellis and K. Tokiroshe: Physs. Rev. C 61 (2000) 015205.

[71] J.L. Goity, D. Lehmann. G. Prezeau and J. Saez: hep-ph/0101011, to appear in Plyys. Lett. B. 
[72] J.L. Gervais and B. Salita: Phys. Rev. Lett 52 (1984) 87; Phys. Rex. D 30 (1984) 1795.

[73] R.F. Dashen, E. Jenkins and A.V. Manohar: Phys. Rev. D 49 (1994) 4713

[74] E. Jenkins: Phys. Rer. D 53 (1996) 2625.

R. Flores-Mendieta. Ch. Hofmann, E. Jenkins and A.V. Manohar: Plurs. Rer. D 62 (2000) 034001.

[75] Th. Hemmert. B. Holstrin and J. Kambor: Phys. Lett. B 395 (1997) 89; .J. Phys. G. 24 (1998) 1831; Phis. Rev. D 55 (1997) 5598.

M. Napsuciale, J.L. Lucio: Nucl. Phys. B 494 (1997) 260.

N. Fettes and U.-G. Meißner: Nucl. Phys. A 679 (2001) 629. 